\title{
Get Rid of Unanimity Rule: The Superiority of Majority Rules with Veto Power
}

\section{Laurent Bouton}

Georgetown University, Centre for Economic Policy Research, and National Bureau of Economic Research

\section{Aniol Llorente-Saguer}

Queen Mary University of London and Centre for Economic Policy Research

\section{Frédéric Malherbe}

London Business School and Centre for Economic Policy Research

\begin{abstract}
We study unanimous decision making under incomplete information. We argue that unanimous decision rules are not all equivalent. We show that majority rules with veto power are (i) Pareto superior to commonly used unanimous rules and (ii) ex ante efficient in a broad class of situations.
\end{abstract}

\section{Introduction}

In many sensitive situations, group decisions are required to be unanimous. For instance, many international organizations could not exist

We thank the coeditor, Jesse Shapiro, and five anonymous referees. We also thank participants of the New Directions in Applied Microeconomics 2013 conference, the Columbia Political Economy Conference 2013, the Priorat Political Economy workshop 2013, the Barcelona Graduate School of Economics PhD Jamboree 2014, the workshop "Theory Meets Experiments" in Zurich, the workshop on Institutions, Individual Behavior, and Economic Outcomes in Alghero, the Fourth Annual Christmas Meeting of Belgian Economists, the Workshop on Information Aggregation in Bonn, the National Bureau of Economic Re-

Electronically published January 16, 2018

[ Journal of Political Economy, 2018, vol. 126, no. 1]

(c) 2018 by The University of Chicago. All rights reserved. 0022-3808/2018/12601-0005\$10.00 
without granting some sort of veto power to their members. In such cases, the constraint stems from sovereignty and enforceability issues (Zamora 1980; Maggi and Morelli 2006; Posner and Sykes 2014). Other examples include partnerships and other unlimited liability companies (Romme 2004) or criminal trials by jury in the United States, where a unanimous verdict is required by the Constitution.

Unanimous decision making can be seen as a means of ensuring that a reform will be adopted only if it constitutes a Pareto improvement over the status quo (Wicksell [1896] 1967; Buchanan and Tullock 1962). However, when information is incomplete, whether or not a reform is adopted also depends on how information is aggregated (Holmstrom and Myerson 1983). The literature focuses on the so-called unanimity rule (henceforth Unanimity), which is commonly used in practice: agents either consent or dissent, and the reform is adopted only if everyone consents. Unfortunately, this rule features poor information aggregation properties (AustenSmith and Banks 1996; Feddersen and Pesendorfer 1998).

We argue that unanimous decision rules are not all equivalent. We show that $k$-Veto rules are (i) Pareto superior to Unanimity and (ii) ex ante efficient in a broad class of situations. Under these rules, agents have three options - consent, dissent, or veto - and the reform is adopted if and only if (i) at least $k$ agents consent and (ii) there is no veto. We refer to this rule as Veto when the quorum $k$ corresponds to that of a simple majority.

Let us sketch the argument that underpins our main results using a simple example. There are three agents who have to vote on whether to adopt a given reform or keep the status quo. The agents have either private or common value, and this is private information. Private-value agents always prefer the status quo. Common-value agents' prior is that the reform can be good or bad with equal probabilities, and they equally dislike a mistake in either direction. Before the vote, each agent receives a private binary signal regarding the merits of the reform. With probability two-thirds, the signal is correct: it is positive if the reform is good and negative if the reform is bad. As a result, the right decision for common-value agents is to adopt the reform if and only if there are at least two positive signals.

search Political Economy and Public Finance Summer Institute, and seminar participants at Ben Gurion University, Boston University, London Business School, London School of Economics, the Max Planck Institute in Bonn, the New Economic School, New York University (New York and Abu Dhabi), Penn State University, Queen Mary, Stanford Graduate School of Business, Technion, University of California Santa Barbara, the University of Haifa, and the University of Warwick. We particularly thank Luca Anderlini, David Austen-Smith, JeanPierre Benoit, Micael Castanheira, Ignacio Esponda, Jean-Guillaume Forand, David Myatt, Roger Myerson, Salvatore Nunnari, Wolfgang Pesendorfer, Oleg Rubanov, Joel Sobel, Richard Van Weelden, and Ro'i Zultan. 
Under any unanimous rule, it is a weakly dominant strategy for privatevalue agents to veto the reform. Therefore, in their presence, the status quo remains, irrespective of common-value agents' behavior. This has two implications. First, common-value agents behave as if there were no private-value agents. Second, unanimous rules' performances can differ only when all agents have common value. Thus, information aggregation is the relevant dimension for welfare comparisons between rules.

Under Unanimity, common-value agents with a bad signal are reluctant to veto the reform because they are pivotal only if the two other agents consent (see Feddersen and Pesendorfer 1998). In equilibrium, when there are no private-value agents, common-value agents make the right decision about 88 percent of the time.

Under Veto, however, it is possible to reveal a negative signal without pinning down the outcome. This is what happens in an equilibrium: commonvalue agents with positive signals consent, and those with negative signals dissent. When there are no private-value agents, common-value agents always make the right decision.

Therefore, Veto Pareto dominates Unanimity in the sense of Holmstrom and Myerson (1983) — both ex ante and interim. That is, whatever their type and signals, all agents (weakly) prefer to use Veto over Unanimity. ${ }^{1}$

When common-value agents have identical preferences (i.e., homogeneous thresholds of reasonable doubt), this result analytically extends to any precision of (possibly biased) signals and any group size. But such a stylized structure of preference is not essential. Indeed, Veto interim Pareto dominates Unanimity in cases in which common-value agents may disagree ex post (i.e., they have heterogeneous thresholds of reasonable doubt).

In our example above, Veto is ex post efficient. Provided that commonvalue agents have homogeneous thresholds, this property generalizes as follows: in all cases, there exists a $k$-Veto rule that enables common-value agents to always make the right decision. When they may disagree ex post, the concept of "right decision" is ambiguous. Ex ante efficiency is then the appropriate benchmark (Holmstrom and Myerson 1983). Relying on a series of numerical examples and asymptotic results, we show that Veto is ex ante efficient in a broad set of situations.

To delimit the applicability of our analysis, it is useful to think in terms of social choice axioms (May 1952). When decisions are required to be unanimous, there is an inevitable tension between neutrality (alternatives

\footnotetext{
${ }^{1}$ When there are only common-value agents, $k$-Veto also Pareto dominates the corresponding $k$-majority rule, strictly when negative signals are sufficiently precise. The reason is that $k$-Veto aggregates information as well as the best among the corresponding $k$-majority rule and Unanimity. See the discussion in Sec. V.B.
} 
are treated equally) and completeness (a decision is made in all cases). We study situations in which a unanimous decision is required to change a well-defined status quo; thus, we give precedence to completeness over neutrality. As a result, our analysis is not relevant to cases such as elections, where neutrality is considered essential (Dasgupta and Maskin 2008).

In real-world situations, the key elements that make our analysis relevant are (i) a common-value dimension, (ii) a unanimity requirement, and (iii) limits to timely and truthful prevote communication. In Section VI, we argue that international organizations are a case in point. We discuss the case of the United Nations Security Council, which illustrates well the reasons for the use of $k$-Veto rules in the real world and why they have replaced Unanimity in some cases.

From a normative point of view, our results suggest that a number of voting bodies that use Unanimity should consider using a $k$-Veto rule instead. Examples include international organizations such as the North Atlantic Treaty Organization, the Council of the European Union in the case of most sensitive topics (a $k$-Veto rule is already in use for matters of common foreign and security policy), and the Southern Common Market (Mercosur). Furthermore, their Pareto dominance, and their relative simplicity, hint that such an institutional reform may not encounter insurmountable resistance.

Finally, there is a vast literature that views trials by jury as an information aggregation problem in which the voting system plays a crucial role. Our analysis applies to such trials if, following this literature, we treat a hung jury as an absence of conviction. One of the main debates focuses on whether to use Unanimity or some form of majority rule. Because Veto combines the strengths of both Unanimity and majority rule, our results may bring the two sides of the debate closer together.

Related literature.-A key idea of this paper is that, compared to Unanimity, Veto enriches the strategic environment so that veto power can be granted without sacrificing information aggregation. This contrasts with an earlier literature that suggests that these two dimensions are in conflict. Specifically, the information aggregation literature shows that the implementation of simple voting rules designed to protect minority rights may lead to poor information aggregation and, somewhat paradoxically, may accomplish neither of the two goals (Feddersen and Pesendorfer 1998).

Later studies have, however, identified cases in which these goals are not incompatible. First, Coughlan (2000) shows that Unanimity aggregates information well in two variations of Feddersen and Pesendorfer's model. In the first, the unanimity requirement is two-sided, but costless mistrials are allowed. Full information aggregation is feasible, but it may require agents to vote again and again on the same reform proposal until it is accepted or rejected unanimously. In the second, he considers pre- 
vote communication. Full information aggregation requires that agents have very similar preferences. In a related paper, Austen-Smith and Feddersen (2006) show that, in setups in which preferences are uncertain, truthful revelation of private information is particularly problematic when agents have veto power. Second, Maug and Yilmaz (2002) show that, when preferences are common knowledge, requiring a majority within all (adequately designed) subgroups of an electorate can achieve information aggregation while protecting minority rights.

Broadly speaking, our paper relates to three strands of the literature. First, it relates to the literature analyzing supermajority rules and/or veto power (e.g., Chen and Ordeshook 1998; Guttman 1998; Groseclose and McCarty 2001; Sobel and Holcombe 2001; Tsebelis 2002; Aghion and Bolton 2003; McGann 2004; Dougherty and Edward 2005; Maggi and Morelli 2006; Dziuda and Loeper 2016; Nunnari 2016). Second, it is connected to the literature that studies information aggregation in two-alternative decisions with strategic voters (see, e.g., Austen-Smith and Banks 1996; Feddersen and Pesendorfer 1996, 1997, 1998; McLennan 1998; Myerson 1998; Chwe 1999; Coughlan 2000; Guarnaschelli, McKelvey, and Palfrey 2000; Maug and Yilmaz 2002; Austen-Smith and Feddersen 2006; Martinelli 2006; Gerardi and Yariv 2007; Battaglini, Morton, and Palfrey 2008, 2010; Van Weelden 2008; Bond and Eraslan 2009; Goeree and Yariv 2011; Mandler 2012; Bhattacharya 2013; McMurray 2013). Third, it is related to the literature studying properties of voting systems (see, e.g., Myerson and Weber 1993; Myerson 2000, 2002; Piketty 2000; Dewan and Myatt 2007; Myatt 2007, 2015; Ahn and Oliveros 2012, 2014; Bouton and Castanheira 2012; Bouton 2013; Ekmekci and Lauermann 2015; Herrera, McMurray, and LlorenteSaguer 2015; Bouton, Castanheira, and Llorente-Saguer 2016).

\section{The Model}

A group of $n \geq 3$ agents (with $n$ odd) must choose between two alternatives, the status quo $Q$ and the reform $R .^{2}$

Information structure.-There are two states of nature, $\omega \in\left\{\omega_{Q}, \omega_{R}\right\}$, which materialize with equal probability. The actual state of nature is not observable, but each agent privately observes an imperfectly informative signal. Conditional on the state of nature, the signals are independently drawn. There are two possible signals: $s_{Q}$ and $s_{R}$. The probability of an agent observing signal $s_{Q}$ is higher in state $\omega_{Q}$ than in state $\omega_{R}$, and the converse is true for $s_{R}$ :

$$
\operatorname{Pr}\left(s_{R} \mid \omega_{R}\right)>\operatorname{Pr}\left(s_{R} \mid \omega_{Q}\right)>0 \quad \text { and } \quad \operatorname{Pr}\left(s_{Q} \mid \omega_{Q}\right)>\operatorname{Pr}\left(s_{Q} \mid \omega_{R}\right)>0 .
$$

This assumption is made without loss of generality. In particular, it allows for cases in which observing one signal is more likely than the other in

${ }^{2}$ That $n$ is odd only simplifies the exposition. 
both states, for example, $\operatorname{Pr}\left(s_{Q} \mid \omega_{R}\right)>\operatorname{Pr}\left(s_{R} \mid \omega_{R}\right)$. In a slight abuse of notation, we denote by $s_{i}$ the signal received by agent $i$.

Preferences.-Agents may have common value or private value. Commonvalue agents all prefer decision $Q$ in state $\omega_{Q}$ and decision $R$ in state $\omega_{R}$. However, common-value agents may differ in their disutility from wrong decisions. We capture this with the following von Neumann-Morgenstern utility function:

\begin{tabular}{lccc}
\hline \hline & \multicolumn{2}{c}{ STATE OF THE WORLD } \\
\cline { 3 - 4 } & & $\omega_{R}$ & $\omega_{Q}$ \\
\hline Group & $R$ & 0 & $-c_{i}$ \\
Decision & $Q$ & $-\left(1-c_{i}\right)$ & 0 \\
\hline
\end{tabular}

where $c_{i} \in(0,1)$. In this representation, $c_{i}$ and $\left(1-c_{i}\right)$ are the respective weight that common-value agent $i$ attaches to errors of type I (adopting a bad reform) and type II (not adopting a good reform). Therefore, $c_{i}$ can be interpreted as a measure of her cautiousness or, in a jury context, her threshold of reasonable doubt. In the baseline model (Secs. III and IV), we focus on the case with homogeneous cautiousness, where $c_{i}$ is the same for all common-value agents. In an extension (Sec. V.A) we study the more general heterogeneous cautiousness cases, where $c_{i}$ may vary across commonvalue agents. Private-value agents prefer the status quo $Q$, irrespective of the state of nature. Each agent faces an ex ante probability $p_{i} \in[0,1)$ of being a private-value agent and a probability $1-p_{i}$ of being a commonvalue one. We denote the corresponding vector by $\mathbf{p} \equiv\left(p_{1}, p_{2}, \ldots, p_{n}\right)$.

Types.-We denote the (realized) type of agent $i$ by $\theta_{i}$, where $\theta_{i}=\theta_{P}$ for private-value agents and $\theta_{i}=\left(s_{i}, c_{i}\right)$ for common-value agents. Thus, the set of possible types is $\Theta \equiv \theta_{P} \cup\left\{s_{Q}, s_{R}\right\} \times(0,1)$. We denote by $\boldsymbol{\theta} \equiv$ $\left(\theta_{1}, \theta_{2}, \ldots, \theta_{n}\right) \in \Theta^{n}$ the vector of realized type profiles in the group.

Mechanisms (or decision rules).-We denote by Ma mechanism that maps a type profile $\theta$ into a group decision (this can be a probabilistic mapping):

$$
M: \Theta^{n} \rightarrow \Delta(\{Q, R\}) .
$$

Voting systems.-A voting system $\Psi$ is a set of possible actions $A^{\Psi}$ and an aggregation rule $d^{\Psi}$ mapping agents' actions into a group decision: $d^{\Psi}$ : $\left\{a \in A^{\Psi}\right\}^{n} \rightarrow\{Q, R\}$. The mapping implied by a voting system and an associated equilibrium strategy profile constitutes a mechanism.

The following voting systems are central to our analysis.

Definition 1. For each $k=1,2, \ldots, n-1$, voting system " $k$-Veto" is defined by $V^{k} \equiv\left\{A^{k}, d^{k}\right\}$, where 


$$
\begin{aligned}
A^{k} & =\{r, q, v\}, \\
d^{k} & =\left\{\begin{array}{l}
R \text { if } X_{v}=0 \text { and } X_{r} \geq k \\
Q \text { otherwise, }
\end{array}\right.
\end{aligned}
$$

where $X_{a}$ denotes the total number of agents playing action $a$.

Since a single $v$ suffices to enforce the status quo $Q$, it can be interpreted as a veto exercised against the reform $R$. Accordingly, $r$ and $q$ can be interpreted as votes for and against the reform. The aggregation rule stipulates that the reform is implemented if, and only if, there is no veto and there are at least $k$ votes in favor of $R$. As such, $k$ can be interpreted as an approval quorum.

Definition 2. Voting system "Unanimity" is defined by $U \equiv\left\{A^{U}, d^{U}\right\}$, where

$$
\begin{aligned}
& A^{U}=\{r, v\}, \\
& d^{U}=\left\{\begin{array}{l}
R \text { if } X_{v}=0 \text { and } X_{r}=n \\
Q \text { otherwise. }
\end{array}\right.
\end{aligned}
$$

Our definition of Unanimity corresponds to the standard in the literature (see, e.g., Feddersen and Pesendorfer 1998; Duggan and Martinelli 2001; Austen-Smith and Feddersen 2006).

REMARK 1. Unanimity is strategically equivalent to a rule often called Consensus. Under Consensus, the reform is adopted if no agent opposes it. Formally, Consensus can be defined by action set $\{q, v\}$ and the same decision rule as under Unanimity. Here, $q$ denotes "silent consent."

Strategy and equilibrium concept.-Following the literature (e.g., Feddersen and Pesendorfer 1998), we focus on responsive symmetric Bayesian Nash equilibria. An agent's strategy is thus a function $\sigma: \Theta \rightarrow \Delta\left(A^{\Psi}\right)$. In particular, $\sigma_{a}(\theta)$ denotes the probability with which an agent of type $\theta$ votes $a$. A responsive profile is such that (i) at least some types play action $r$ with positive probability, and (ii) not all of them play $r$ with probability one. This ensures that, in equilibrium, some pivot probabilities are strictly positive; that is, agents affect the outcome of the vote with positive probability. Given that the reform $R$ is implemented with positive probability for any responsive strategy profile, private-value agents always strictly prefer to vote against $R$. Thus, they always use their veto under any unanimous rule. Henceforth, we simply refer to responsive symmetric equilibria as equilibria.

\section{Equilibrium Analysis}

In this section, we present the main equilibrium results and provide intuition that will be useful to understand the welfare results. 
Any equilibrium under $k$-Veto satisfies two conditions:

Proposition 1. For any $\mathbf{p}, \sigma^{*}$ is an equilibrium under $k$-Veto if and only if

i. private-value agents veto the reform, and

ii. $\sigma^{*}$ is an equilibrium of the corresponding game with $\mathbf{p}=\mathbf{0}$.

Proof. First, recall that $v$ is a strictly dominant strategy for $\theta_{P}$-agents. Therefore, any equilibrium under $k$-Veto satisfies point $i$ in the proposition. Second, given point $\mathrm{i}$, if there exists $i$ such that $\theta_{i}=\theta_{P}$, then the group decision is $Q$ irrespective of what any other agent does. Thus, commonvalue agents condition their behavior on $\theta_{i} \neq \theta_{P}$ for all $i$. Therefore, we have that (1) the strategy profile played by common-value agents in any equilibrium under $k$-Veto must form an equilibrium when $\mathbf{p}=\mathbf{0}$, and (2) if $\sigma^{*}$ is an equilibrium strategy profile of the game when $\mathbf{p}=\mathbf{0}$, it must also be an optimal strategy profile for common-value agents for all $\mathbf{p} \neq \mathbf{0}$. QED

On the basis of this result, we focus on the pure common-value game $(\mathbf{p}=\mathbf{0})$ to describe the behavior of common-value agents.

For the remainder of this section, we assume that $c_{i}$ is identical for all common-value agents. For simplicity, we set $c_{i}=1 / 2{ }^{3}$ At this point it is useful to introduce new pieces of notation. First, we denote the (relative) precision, or likelihood ratio, of signal $s_{Q}$ by

$$
\phi_{Q} \equiv \frac{\operatorname{Pr}\left(s_{Q} \mid \omega_{Q}\right)}{\operatorname{Pr}\left(s_{Q} \mid \omega_{R}\right)} \in(1, \infty)
$$

and that of signal $s_{R}$ by

$$
\phi_{R} \equiv \frac{\operatorname{Pr}\left(s_{R} \mid \omega_{R}\right)}{\operatorname{Pr}\left(s_{R} \mid \omega_{Q}\right)} \in(1, \infty) .
$$

Second, with a slight abuse of notation, we denote by $\theta_{R}\left(\theta_{Q}\right)$ the type of a common-value agent who receives signal $s_{R}\left(s_{Q}\right)$.

The following lemma extends Austen-Smith and Banks's (1996) result on informative voting under $k$-majority rules to our $k$-Veto system.

Lemma 1. For each tuple $\left(\phi_{Q}, \phi_{R}, n, \mathbf{p}\right)$, there is a $k$ such that $k$-Veto admits an equilibrium in which (i) $\theta_{R}$-agents play $r$ and (ii) $\theta_{Q}$-agents play $q$ if $\phi_{Q}<\left(\phi_{R}\right)^{n-1}$, and $v$ otherwise.

Proof. See appendix C.

We now describe equilibrium behavior in greater detail for Veto.

Lemma 2. Under Veto, the pure common-value game admits at most two equilibria.

Proof. The full characterization of the set of equilibria and the proofs are in appendix A.

\footnotetext{
${ }^{3}$ Given that we consider all possible signal precision, this assumption is without loss of generality.
} 
We now discuss the two possible equilibria in turn.

The information-aggregation equilibrium.- In the first and most interesting equilibrium, agent behavior is easily understood through a series of simple examples.

First, assume that signal precision is symmetric. That is, $\phi_{R}=\phi_{Q}$. In this case, $\theta_{R}$-agents simply play $r$ and $\theta_{Q}$-agents play $q$. The reason is that both signals have the same information content and the aggregation rule gives the same weight to $r$ and $q$ votes.

If signal precision is sufficiently asymmetric, agents "compensate"; that is, agents with the less informative signal mix between $r$ and $q$. As long as $\phi_{Q}$ is not too high (compared to $\phi_{R}$ ), agents do not use their veto power and their equilibrium behavior is exactly the same as what it would be under simple majority rule. However, when $\phi_{Q}$ exceeds a certain threshold, agents with an $s_{Q}$ signal start vetoing with positive probability.

Finally, if $\phi_{Q} \geq\left(\phi_{R}\right)^{n-1}$, the equilibrium under Veto is unique (and it is the same as the unique equilibrium under Unanimity). In that case, the signal $s_{Q}$ is so precise that it becomes a weakly dominant strategy for $\theta_{Q^{-}}$ agents to veto (in fact, conditional on observing all the signals, a single negative signal suffices to convince an agent that the reform is bad). The best response for $\theta_{R}$-agents is to vote $r$, and the reform is implemented if and only if there is no $\theta_{Q}$-agent.

REMARK 2. In the information-aggregation equilibrium of the general game, agent behavior can be interpreted as a combination of what the agent would do under Unanimity and majority rule (without veto power). Veto indeed allows agents to reproduce any strategy played under majority rule or Unanimity. In particular, they use the veto power to protect their private interest (which they cannot do under majority rule), and they vote against the reform (without vetoing it) when they have a negative, but nonconclusive, signal about it (which they cannot do under Unanimity).

The Unanimity-like equilibrium.-When $\phi_{Q}<\left(\phi_{R}\right)^{n-1}$, Veto admits a second equilibrium. This equilibrium corresponds to the unique equilibrium under Unanimity, as characterized in Feddersen and Pesendorfer (1998): $\theta_{R}$-agents vote $r$, and $\theta_{Q^{-}}$agents randomize between $r$ and $v$.

We see the Unanimity-like equilibrium as a less credible predictor of agent behavior than the information aggregation equilibrium (see the discussion in app. A and Bouton, Llorente-Saguer, and Malherbe [2017]). If agents play the former, outcomes are simply equivalent under Veto and Unanimity, and the two systems feature identical welfare properties. This is why the welfare analysis focuses on the information aggregation equilibrium.

\section{Welfare Analysis}

In this section, we study the welfare properties of $k$-Veto based on the equilibrium analysis above (i.e., when common-value agents have homo- 
geneous cautiousness: $c_{i}=1 / 2$ ). We first establish that Veto interim dominates Unanimity. We then show that (i) Veto is ex ante efficient when agents play pure strategies in equilibrium, (ii) there always exists a $k$-Veto system that is efficient, and (iii) Veto is asymptotically optimal.

\section{A. Efficiency Concepts}

Utility.-For a given mechanism $M$ and a given profile $\theta$, we can define $e_{1}(M, \theta)$ and $e_{2}(M, \theta)$, the probability that decision $R$ is made in state $\omega_{Q}$, and the probability that decision $Q$ is made in state $\omega_{R}$, respectively. Therefore, they correspond to (ex post) expected probabilities of errors of type I and II (from a common-value agent's perspective). We have

$$
\begin{aligned}
& e_{1}(M, \boldsymbol{\theta}) \equiv \operatorname{Pr}\left(\omega_{Q} \mid \boldsymbol{\theta}\right) \operatorname{Pr}(M(\boldsymbol{\theta})=R), \\
& e_{2}(M, \boldsymbol{\theta}) \equiv \operatorname{Pr}\left(\omega_{R} \mid \boldsymbol{\theta}\right) \operatorname{Pr}(M(\boldsymbol{\theta})=Q) .
\end{aligned}
$$

We are now in a position to define the ex post, interim, and ex ante utility of an agent $i$, under any mechanism $M$ :

$$
\begin{aligned}
u_{i}(M \mid \theta) & \equiv-c_{i} e_{1}(M, \theta)-\left(1-c_{i}\right) e_{2}(M, \theta), \\
u_{i}\left(M \mid \theta_{i}\right) & \equiv E_{\theta}\left[u_{i}(M \mid \theta) \mid \theta_{i}\right], \\
u_{i}(M) & \equiv E_{\theta}\left[u_{i}(M \mid \theta)\right] .
\end{aligned}
$$

Dominance and efficiency.-We focus on Pareto dominance and efficiency as formalized by Holmstrom and Myerson (1983).

Definition 3. Mechanism $M$ interim dominates mechanism $M^{\prime}$ if $u_{i}\left(M \mid \theta_{i}\right) \geq u_{i}\left(M^{\prime} \mid \theta_{i}\right)$, for all $i, \theta_{i}$. And $M$ interim strictly dominates $M^{\prime}$ if this condition also holds with strict inequality for some $i$ and $\theta_{i}$.

Ex ante and ex post dominance are defined similarly, and the definitions of efficiency directly follow.

Definition 4. A mechanism is ex ante (interim, ex post) efficient if it is not ex ante (interim, ex post) strictly dominated by another mechanism.

Note that interim dominance implies ex ante dominance and that ex ante efficiency implies interim efficiency.

Comparing voting systems. - A voting system $\Psi$ and an associated strategy profile $\sigma$ form a mechanism that maps realized type profiles into group decisions. We denote such a mechanism $\Psi_{\sigma}$ :

$$
\Psi_{\sigma}: \Theta^{n} \rightarrow \Delta(\{Q, R\}) .
$$

For what follows, let us adapt the definition of interim dominance and take into account potential equilibrium multiplicity.

Definition 5. Voting system $\Psi$ interim dominates voting system $\Psi^{\prime}$ if there exists an equilibrium $\sigma$ under $\Psi$ such that $u_{i}\left(\Psi_{\sigma} \mid \theta_{i}\right) \geq u_{i}\left(\Psi_{\sigma^{\prime}}^{\prime} \mid \theta_{i}\right)$ for 
all equilibria $\sigma^{\prime}$ under $\Psi^{\prime}$, for all $i$, and all $\theta_{i}$. And $\Psi$ interim strictly dominates $\Psi^{\prime}$ if, in each $\sigma^{\prime}$, this condition also holds with strict inequality for some $i$ and some $\theta_{i}$.

\section{B. Veto Interim Dominates Unanimity}

Theorem 1. For all tuples $\left(\phi_{Q}, \phi_{R}, n, \mathbf{p}\right)$, Veto interim (and thus ex ante) dominates Unanimity, strictly unless $\phi_{Q} \geq\left(\phi_{R}\right)^{n-1}$.

Proof. See appendix C.

Theorem 1 is a powerful result: it implies that no agent (even after learning their type) would object to getting rid of Unanimity and using Veto instead.

The intuition is as follows. First, when there is at least one private-value agent in the group, the status quo is kept under the two systems. Let us focus on the cases in which there are only common-value agents and consider $\theta_{Q^{-}}$and $\theta_{R}$-agents in turn. Under Unanimity, $\theta_{Q^{-}}$agents play $v$ with positive probability. Thus, their interim utility equals the utility of getting $Q$ with probability one. Since they can also play $v$ under Veto (and get $Q$ with probability one), they cannot be worse off. By a simple revealed preference argument, they are strictly better off in the cases in which they strictly prefer to vote $q$ (which is typical of the information aggregation equilibrium).

For $\theta_{R}$-agents, the intuition goes as follows: under Unanimity the reform $R$ is rarely chosen. This implies that the probability of making a mistake is relatively high in state $\omega_{R^{*}}$ Given that $\theta_{R}$-agents believe that state $\omega_{R}$ is more likely than state $\omega_{Q}$, their interim utility under Unanimity is low. Since Veto does not suffer from the same weakness, $\theta_{R}$-agents are strictly better off under Veto than under Unanimity.

One can also establish that the interim dominance of $k$-Veto over Unanimity implies the interim dominance of $k^{\prime}$-Veto over Unanimity for $k<$ $k^{\prime} \leq n$. To see the intuition behind this, pick an arbitrary vector of values for $\phi_{Q}, \phi_{R}, n$, and $\mathbf{p}$ and denote $k^{*}$ the quorum such that $k^{*}$-Veto is efficient (see theorem 2 below). If $k^{\prime}>k^{*}$, the quorum is too high to aggregate information perfectly. However, under Unanimity, the corresponding quorum would be $n$, and information aggregation would be even worse. Now, consider $k^{\prime}<k^{*}$. In this case, the quorum is too low. But we know that Veto dominates when $k=(n+1) / 2$. Therefore, increasing the quorum (i.e., bringing it closer to $k^{*}$ ) can only improve information aggregation. One cannot conclude, however, that the strict dominance result applies for all parameter values for all quorums strictly below $(n+1) / 2$.

\section{Is Veto Efficient?}

We have established that Veto dominates Unanimity, but are there mechanisms that dominate Veto? 
Given our research question, we restrict our attention to unanimous mechanisms, that is, mechanisms that allow any agent to enforce the status quo. In a voting setup, this means that agents must have veto power. In a more general mechanism design approach, we capture this restriction by imposing that, under an admissible mechanism, the interim utility of all agents is at least as high as their utility under the status quo.

This constraint (which we will refer to as the veto constraint) can be understood as an interim participation constraint, where agents' outside option is their utility under the status quo. The veto constraint can equally be interpreted as resulting from an ex ante participation constraint in a version of the model in which some agents in some states would have a sufficiently large disutility from the reform. In this case, agents are willing to participate ex ante only if the mechanism ensures that they will be able to block any reform that would critically hurt their interest. ${ }^{4}$

Definition 6. A mechanism is admissible if it satisfies

$$
u_{i}\left(M \mid \theta_{i}\right) \geq u_{i}\left(M_{Q} \mid \theta_{i}\right) \quad \forall i,
$$

where $M_{Q}$ is a trivial mechanism that keeps the status quo for all type profiles: $M_{Q}(\theta)=Q$ for all $\boldsymbol{\theta}$. We denote by $\mathcal{M}$ the set of such admissible mechanisms.

Since private-value agents dislike the reform irrespective of the state of nature, all admissible mechanisms must keep the status quo for any type profile including a private-value agent. This implies that a mechanism's relative performance (and its efficiency) can be assessed on the basis of its outcome for type profiles including only common-value agents.

Henceforth, we will compare only admissible mechanisms. For instance, when we state that a mechanism is efficient, this must be understood as efficient within the set of admissible mechanisms. When relevant, we also impose incentive compatibility (see Sec. V.A). Given that $k$-Veto and Unanimity give veto power to each agent in the group (and that incentive compatibility constraints are, by definition, satisfied in equilibrium), both voting systems at equilibrium are admissible mechanisms.

Lemma 3. The following mechanism is ex ante efficient:

$$
M^{*}(\boldsymbol{\theta}) \equiv\left\{\begin{array}{l}
R \text { if } \forall i, \theta_{i} \neq \theta_{P} \text { and } \operatorname{Pr}\left(\omega_{R} \mid \boldsymbol{\theta}\right) \geq c_{i} \\
Q \text { otherwise. }
\end{array}\right.
$$

Proof. First, note that $M^{*}(\theta)$ is admissible. Second, since there is no ex post disagreement among common-value agents, it is ex post efficient. Third, there is no admissible mechanism that gives higher ex post utility to any agent. Thus, it is incentive compatible and ex ante efficient. QED

\footnotetext{
${ }^{4}$ Such an interpretation directly speaks to applications such as partnerships or to sovereignty issues in the case of international organizations.
} 
Definition 7. Voting system $\Psi$ is efficient if there exists an equilibrium $\sigma$ under $\Psi$ such that $\Psi_{\sigma}(\boldsymbol{\theta})=M^{*}(\boldsymbol{\theta})$ for all $\boldsymbol{\theta}$.

Proposition 2. For all $\mathbf{p}$, Veto is ex ante efficient if

$$
\phi_{Q} \in\left[\left(\phi_{R}\right)^{(n-1) /(n+1)},\left(\phi_{R}\right)^{(n+1) /(n-1)}\right]
$$

or $\phi_{Q} \geq\left(\phi_{R}\right)^{n-1}$.

Proof. The only nontrivial case is one in which the type profile does not include private-value agents, that is, $\theta_{i} \neq \theta_{P}$ for all $i$. Consider the information-aggregation equilibrium characterized in proposition 6 (in app. A). If

$$
\phi_{Q} \in\left[\left(\phi_{R}\right)^{(n-1) /(n+1)},\left(\phi_{R}\right)^{(n+1) /(n-1)}\right],
$$

$\theta_{R}$-agents play $r$ and $\theta_{Q}$-agents play $q$, and $R$ is chosen if and only if there are more $r$-votes than $q$-votes. However, given that

$$
\phi_{Q} \in\left[\left(\phi_{R}\right)^{(n-1) /(n+1)},\left(\phi_{R}\right)^{(n+1) /(n-1)}\right],
$$

$\operatorname{Pr}\left(\omega_{R} \mid \boldsymbol{\theta}\right)>1 / 2$ if and only if there are more $\theta_{R}$-agents than $\theta_{Q^{-}}$-agents. If $\phi_{Q} \geq\left(\phi_{R}\right)^{n-1}, \theta_{R}$-agents play $r$ and $\theta_{Q}$-agents play $v$; $R$ is thus implemented only if there are no $\theta_{Q}$-agents. But given that $\phi_{Q} \geq\left(\phi_{R}\right)^{n-1}, \operatorname{Pr}\left(\omega_{R} \mid \boldsymbol{\theta}\right)>1 / 2$ if and only if there are no $\theta_{Q}$-agents. Therefore, Veto at the informationaggregation equilibrium matches $M^{*}(\boldsymbol{\theta})$ for all $\boldsymbol{\theta}$. QED

For a voting system to be efficient, it is necessary that agents use pure strategies (otherwise one cannot have full information revelation). However, we know from lemma 1 that adjusting the approval quorum $k$ can induce agents to do so. This leads to the following theorem. ${ }^{5}$

Theorem 2. For each tuple $\left(\phi_{Q}, \phi_{R}, n, \mathbf{p}\right)$, there exists a $k$-Veto voting system that is ex ante efficient.

Proof. If $\phi_{Q} \geq\left(\phi_{R}\right)^{n-1}$, all $k$-Veto rules are efficient. If $\phi_{Q}<\left(\phi_{R}\right)^{n-1}$, we know from lemma 1 that, under $k$-Veto, for each tuple $\left(\phi_{Q}, \phi_{R}, n, \mathbf{p}\right)$, there is a $k$ such that the rule admits an equilibrium in which $\theta_{R}$-agents play $r$ and $\theta_{Q^{-}}$agents play $q$. This directly implies that, for such a $k, k$-Veto implements $R$ if and only if there are no private-value agents and the number of $s_{R}$ is greater than or equal to $k$. Given lemma 3 , it remains to be proven that $\operatorname{Pr}\left(\omega_{R} \mid \boldsymbol{\theta}\right) \geq 1 / 2$ if and only if the number of $s_{R}$ signals is greater than or equal to $k$. But for $\theta_{R}$-agents to play $r$ and $\theta_{Q}$-agents to play $q$ in equilibrium, it must be the case that (i) for any $\theta$ such that the number of $s_{R}$ received equals $k, \operatorname{Pr}\left(\omega_{R} \mid \boldsymbol{\theta}\right) \geq 1 / 2$, and (ii) for any $\boldsymbol{\theta}$ such that the number of $s_{R}$ signals received is equal to $k-1,1 / 2>\operatorname{Pr}\left(\omega_{R} \mid \boldsymbol{\theta}\right)$. QED

${ }^{5}$ This result is related to theorem 1 in Costinot and Kartik (2007). They show that, in the standard framework of the Condorcet jury literature with binary states and binary signals, there is a majority rule that is ex ante efficient. In our setup, however, majority rules are not admissible (except when $\mathbf{p}=\mathbf{0}$ ). 
In practice, it would be useful to have a system that performs well for different sets of parameters. This is, for instance, the case for a decision body that presides over many different issues but cannot adapt its voting system to the question at hand. Unfortunately, there is no $k$-Veto voting system that is ex ante efficient for all values of the parameters. However, as implied above, Veto's departure from efficiency stems from the use of mixed strategies. Therefore, increasing the group's size reduces inefficiency. As we show in the next section, it vanishes as $n$ increases.

\section{Asymptotic Results}

In this subsection, we establish Veto's appealing asymptotic properties.

Definition 8. Mechanism $M$ is asymptotically optimal if, with a probability that tends to one when $n$ tends to infinity, it selects $R$ if $\omega=\omega_{R}$ and $\theta_{i} \neq \theta_{P}$ for all $i$, and $Q$ otherwise.

The decision is optimal if, given the state of nature and the type profile, the reform is adopted if and only if it constitutes a Pareto improvement over the status quo. ${ }^{6}$

Let $p_{\text {lim }}$ denote the limit probability of having at least one private-value agent in the group: ${ }^{7}$

$$
p_{\lim } \equiv 1-\lim _{n \rightarrow \infty} \prod_{i=1}^{n}\left(1-p_{i}\right) .
$$

When $p_{\text {lim }}=1$, the optimal decision is always to keep the status quo. In that case, all admissible mechanisms are asymptotically optimal. When $p_{\text {lim }}<1$, information aggregation remains relevant in the limit. It turns out that Veto is asymptotically optimal in this case as well.

Proposition 3. For all $\phi_{Q}, \phi_{R}$, and $\mathbf{p}$, Veto is asymptotically optimal.

Proof. First, given that $\sigma_{v}\left(\theta_{P}\right)=1$, we have that for all $\theta$ such that $\theta_{i}=$ $\theta_{P}$ for some $i$, Veto keeps the status quo $Q$, which is optimal. Second, it is easy to see that in the information-aggregation equilibrium of Section III, for any $\phi_{R}$ and $\phi_{Q}$, in the limit, $\sigma_{v}(\theta)=0$ for all $\theta \in\left\{\theta_{R}, \theta_{Q}\right\}$. Therefore, if $\theta_{i} \neq \theta_{P}$ for all $i$, the outcome under Veto is exactly the same as under simple majority rule when $\mathbf{p}=\mathbf{0}$. We know from Feddersen and Pesendorfer (1998) that, when $\mathbf{p}=\mathbf{0}$, simple majority rule aggregates information perfectly in the limit; that is, the group chooses $R$ in state $\omega_{R}$ and $Q$ in state $\omega_{Q}$ with a probability that tends to one when $n \rightarrow \infty .{ }^{8} \mathrm{QED}$

In the case in which $p_{\lim }<1$, Unanimity is not asymptotically optimal when it is interim strictly dominated by Veto.

\footnotetext{
${ }^{6}$ For Veto and Unanimity, this definition of asymptotic optimality corresponds to the full information equivalence benchmark used in the Condorcet jury theorem literature.

7 The limit exists, since $\Pi_{i=1}^{n}\left(1-p_{i}\right)$ monotonically decreases with $n$ and is bounded below by zero.

${ }^{8}$ Although our setup is slightly different from that of Feddersen and Pesendorfer (1998), the proof is almost identical.
} 


\section{Discussion and Extensions}

In this section, we first extend our analysis to a version of the model that allows for disagreement among common-value agents. Then we explore the consequences of relaxing the veto constraint.

\section{A. Preference Diversity}

To introduce disagreement among common-value agents, we allow $c_{i}$ to differ across them. However, to avoid unnecessary complications, we assume that the probability of receiving the correct signal is the same in both states, that is, $\operatorname{Pr}\left(s_{Q} \mid \omega_{Q}\right)=\operatorname{Pr}\left(s_{R} \mid \omega_{R}\right)=\pi>1 / 2$.

We adopt a twofold strategy to overcome the technical challenges heterogeneous $c_{i}$ 's imply. First, we focus on a case with three agents and two levels of cautiousness. In this case, the model is still analytically tractable: we are able to fully characterize equilibria and, for some values of the parameters, establish the welfare results analytically. We use numerical methods otherwise.

Second, we study the asymptotic properties of Veto. This is the standard approach for analyzing models with rich preference structure, signal space, and/or state space. We show that Veto is still asymptotically optimal (and thus interim dominates Unanimity when $n$ is sufficiently large).

\section{Three Agents}

There are three common-value agents that may differ in their level of cautiousness. ${ }^{9}$ Agents can be neutral, in which case they have the same cautiousness parameter as before $\left(c_{N}=1 / 2\right)$, or cautious, with $c_{H} \in$ $(1 / 2,1)$. That is, cautious agents dislike errors of type I (adopting a bad reform) relatively more than errors of type II (not adopting a good reform). Ex ante, agents face an identical probability $\rho \in[0,1)$ of being cautious.

Example.-To provide intuition on agent behavior and why Veto still dominates Unanimity, we first focus on parameter values for which there is a pure strategy equilibrium under Veto. Suppose that (i) $\rho$ is not too high (see below for details), and (ii) cautious agents, if they could observe all signals, prefer the reform only if there are three signals $s_{R}$ (by construction, neutral agents prefer the reform if there are at least two signals $s_{R}$ ).

Under Veto, there is an equilibrium such that cautious agents vote $q$ if they receive a signal $s_{R}$, and they veto if they receive a signal $s_{Q}$; neutral agents vote $r$ if they receive a signal $s_{R}$, and they vote $q$ if they receive a signal $s_{Q}$. Under Unanimity, if $c_{H}$ is high enough, cautious agents veto ir-

\footnotetext{
${ }^{9}$ We do not include private-value agents because this would not affect the results.
} 
respective of their signal. Neutral agents vote $r$ if they receive a signal $s_{R}$, and they mix between $r$ and $v$ with a signal $s_{Q}$.

Why does Veto interim dominate Unanimity? First, if there are only neutral agents, Veto aggregates information perfectly (which leads to the right decision from their common viewpoint), but Unanimity does not. Second, if there is at least one cautious agent, (i) under Unanimity, the status quo always remains; and (ii) under Veto, the status quo remains except in the case in which all agents receive an $s_{R}$ signal and at most one of them is cautious. But when that is the case, $R$ is the only ex post efficient, and therefore "right," decision. Thus, Veto strictly interim dominates Unanimity.

Generalization.-We can characterize an equilibrium for all values of the parameters under both Veto and Unanimity. The example above corresponds to

$$
c_{H} \in\left(\frac{\pi^{2}}{\pi^{2}+(1-\pi)^{2}}, \frac{\pi^{3}}{\pi^{3}+(1-\pi)^{3}}\right)
$$

and $\rho \leq 1 / 2$. On the basis of this characterization, we are able to analytically prove that Veto interim dominates Unanimity for two other sets of parameter values: if $c_{H} \leq \pi$ or $c_{H} \geq \pi^{3} /\left[\pi^{3}+(1-\pi)^{3}\right]$. In both cases, the intuition from the baseline model is useful: when $c_{H} \leq \pi$, cautious agents are essentially neutral agents because there is no ex post disagreement; when $c_{H} \geq \pi^{3} /\left[\pi^{3}+(1-\pi)^{3}\right]$, it is a weakly dominant strategy for cautious agents to veto the reform irrespective of their signal. Thus, they behave like private-value agents. The interim dominance result of theorem 1 therefore readily extends.

For other parameter values, tractability is an issue. We use the following numerical approach. First, we generate a grid for $c_{H} \in[1 / 2,1], \rho \in$ $(0,1)$, and $\pi \in(1 / 2,1)$ in steps of 0.001 . Second, for each parameter combination, we compute interim utility ( $u p$ to a precision of $1 \mathrm{E}^{-10}$ ) for each type of agent under both systems based on our analytical characterization of equilibrium. Veto both interim and ex ante strictly dominates Unanimity for all parameter combinations in the grid.

Efficiency.-When common-value agents may disagree ex post, incentive compatibility constraints must be taken into account. For each point of the parameter grid, we consider all pure mechanisms - that is, all possible mappings from type profiles into pure decisions $\left(M: \Theta^{n} \rightarrow\{R, Q\}\right)$. For each of these mechanisms, we compute interim utility $u_{i}\left(M \mid \theta_{i}\right)$ under truthful revelation and discard those that are not admissible or incentive compatible. Our efficiency benchmark $M_{I C}^{*}$ is the mechanism that maximizes ex ante utility among the remaining candidates.

Three major patterns emerge (see the online appendix for more details). First, Veto matches $M_{I C}^{*}$ when $c_{H}$ takes either low or high values. 
In contrast, Unanimity is always ex ante strictly dominated by $M_{I C}^{*}$. Second, for intermediate values of $c_{H}$, Veto does not match $M_{I C}^{*}$, but it generates fairly close levels of ex ante utility. For a certain range of parameters, Veto even strictly ex ante dominates $M_{I C}^{*} \cdot{ }^{10}$ Finally, when Veto is ex ante strictly dominated by $M_{I C}^{*}$, Unanimity is dominated by $M_{I C}^{*}$ by a margin that is typically an order of magnitude larger (when both $\rho$ and $c_{H}$ are large, however, this margin decreases).

\section{Large Groups}

To explore the asymptotic properties of Veto (and Unanimity) and allow for private-value agents, we adapt the setup of Gerardi (2000). With probability $p_{i} \in[0,1)$, agent $i$ is a private-value agent $\left(\theta_{i}=\theta_{P}\right)$. With probability $1-p_{i}$, agent $i$ has common value with cautiousness $c_{i}$ drawn from a probability distribution $F$ with support on the interval $(\underline{c}, \bar{c})$, with $0 \leq$ $\underline{c}<\pi<\bar{c}<1 .{ }^{11}$ As in Gerardi (2000), we assume that $F$ is continuous, is strictly increasing, and admits a density $f$, such that $\lim _{x \rightarrow \underline{c}} f(x)>0$ and $\lim _{x \rightarrow \bar{c}} f(x)>0$. Our definition of asymptotic optimality (definition 8) still applies.

The main advantage of considering a setup (almost) identical to that of Gerardi (2000) is that we can use his results about nonunanimous rules in our proof of the following result.

Proposition 4. For all $\phi_{Q}, \phi_{R}$, and $\mathbf{p}$, Veto is asymptotically optimal.

Proof. See the online appendix.

As in the baseline model, Veto gives private-value agents the power to enforce the status quo without affecting the behavior of common-value agents. The crux of the matter is to understand why the most cautious common-value agents (i.e., those with a large $c_{i}$ ) do not want to use their veto power.

When other common-value agents play the equilibrium strategy under majority rule, the expected outcome is $R$ in state $\omega_{R}$ and $Q$ in state $\omega_{Q}$. Therefore, by vetoing the reform, a common-value agent is more likely to prevent a desirable reform than an undesirable one. As the size of the group grows larger, the relative likelihood of a mistake tends to infinity, whence no common-value agent wants to veto the reform.

In our setup, we can show that an asymptotically optimal mechanism interim strictly dominates any mechanism that is not asymptotically optimal. On the basis of this, we get the following result.

${ }^{10}$ This is possible when the equilibrium is in mixed strategies.

${ }^{11}$ In Gerardi (2000), $\bar{c}=1$. In our context, this case is somehow extreme (and unnecessarily complicates the analysis because of an order-of-limits issue). When $\bar{c}=1$, there may be distribution functions such that the probability of having an agent who behaves as a private-value agent (because she has very high cautiousness) tends to one when $n$ tends to infinity. In these particular cases, Veto and Unanimity would then both be asymptotically optimal. 
Proposition 5. For all $\phi_{Q}, \phi_{R}$, and $\mathbf{p}$, for $n$ sufficiently large, Veto interim dominates Unanimity.

Proof. See the online appendix.

\section{B. Nonunanimous Decision Making}

Our analysis focuses on information aggregation when there is a veto constraint. In many of the applications we have in mind, this constraint emerges from the need to protect single individuals (or states). Thus, the veto constraint can be interpreted as an extreme need for minority protection. But what if the minority in question is larger than one?

Minority larger than one.-Consider a group of $n$ agents whose objective is to implement the reform if and only if two conditions are satisfied: (i) it is against the private interests of no more than $f$ agents, and (ii) the reform is good (from the common-value agent standpoint). Which voting system should they use?

Veto achieves this objective for $f=0$. We conjecture that the following simple rules would achieve this objective for $f>0$ (because, similarly to $k$-Veto, they make it easier for agents to dissociate the minority protection dimension from the information aggregation dimension). Under these rules, the action set is the same as under Veto (i.e., $\{r, q, v\}$ ), and the aggregation rule is

$$
d^{f}=\left\{\begin{array}{l}
R \text { if } X_{v} \leq f \text { and } X_{r} \geq \frac{n+1}{2} \\
Q \text { otherwise. }
\end{array}\right.
$$

That is, the reform is adopted if and only if no more than fagents vote $v$ and a majority vote $r$. Here, $v$ is a strong action against the reform, but it is no longer a veto.

If these rules indeed show properties similar to those of Veto, this would provide an argument for the so-called filibuster procedure, which exists in many parliamentary systems, as a way to balance minority protection and information aggregation. ${ }^{12}$

Veto versus majority rules.- Now imagine that there is no minority to protect. For instance, consider a pure common-value setup (i.e., $\mathbf{p}=\mathbf{0}$ ). Can we say something of the comparison of Veto and majority rule? The answer is yes: Veto ex ante dominates majority rule (strictly when $v$ is played with positive probability in the information-aggregation equilibrium un-

\footnotetext{
${ }^{12}$ We are very grateful to an anonymous referee for suggesting this extension and its application to the filibuster procedure.
} 
der Veto). ${ }^{13}$ To understand why Veto dominates majority rule, first note that any strategy profile under majority rule can be reproduced under Veto. Second, recall from McLennan (1998) that in a pure commonvalue environment, a strategy profile producing the maximal ex ante utility must be an equilibrium. Therefore, there always exists an equilibrium under Veto that produces an ex ante utility at least as high as in the unique equilibrium under majority rule. However, Veto has a larger action set, which proves useful in cases in which the negative signal is precise enough. These are the cases in which Veto strictly dominates majority rule.

\section{Empirical Relevance and Applications}

In this section, we discuss the empirical relevance of our analysis. First, we argue that international organizations often combine an information aggregation problem in a common-value environment with a veto constraint and factors that limit timely and truthful communication. ${ }^{14}$ To argue this point, we discuss the UN Security Council. It is particularly relevant because it suggests that using Veto instead of Unanimity does indeed improve outcome efficiency. We then extend the argument to international organizations in general.

Second, we discuss our results in the context of a vast literature that views trials by jury as an information aggregation problem in which the voting system plays a crucial role. One of the main policy debates focuses on whether to use Unanimity or some form of majority rule. We argue that our results may bring the two sides of this debate closer together.

\section{A. International Organizations}

The Charter of the United Nations is explicit about the common-value dimension: it proclaims that the peoples of the United Nations resolve to combine their efforts to accomplish common aims (international peace, economic and social advancement of all people, etc.). It grounds these aims in the ideals of justice and fundamental human rights.

The charter also states that the Security Council shall make decisions based on an affirmative vote of nine members, which must include the

\footnotetext{
${ }^{13}$ See proposition 10 in the online appendix. This is related to the result of Duggan and Martinelli (2001) that unanimity rule can dominate nonunanimous rules for some structures of information.

${ }^{14}$ In our model, when $c_{i}^{\text {'s }}$ are identical, if prevote communication is allowed and costless, there exist efficient equilibria in which voters truthfully reveal their types in the communication stage and vote for the optimal group decision in the voting stage (this is a trivial extension of proposition 8 in Coughlan [2000], in which we allow agents to communicate their type). In that case, we can simply think of $k$-Veto as a method of formalizing information aggregation that might otherwise be accomplished through communication.
} 
concurring votes of the permanent members. This, in effect, gives veto power to each permanent member. However, as noted on the UN website, "If a permanent member does not fully agree with a proposed resolution but does not wish to cast a veto, it may choose to abstain, thus allowing the resolution to be adopted if it obtains the required number of nine favorable votes." With the exception that only five countries have veto power, this corresponds to a $k$-Veto rule, with an approval quorum ( $k$ ) of nine out of 15 members. Indeed, the 10 elected members can vote yes, no, or abstain. The five permanent members can vote yes, veto, or $a b$ stain. In both cases, abstention counts as a no.

The origin of veto power for the permanent members has been linked to their desire to protect their sovereignty (see, e.g., Reston 1946; Lee 1947; Posner and Sykes 2014) and to implementability concerns (see, e.g., Winter 1996). The sovereignty issue is well illustrated by President Truman in his memoirs. He wrote, "In the present world setup sovereign powers are very jealous of their rights. We had to recognize this as a condition and to seek united action through compromise" $(1965,311)$. On implementability concerns, Winter $(1996,813)$ writes, "The idea of granting permanent members veto power evolved directly from the fact that the enforcement of many Security Council resolutions would require the military and financial support of the superpowers. Hence, without the unanimous consent of the permanent members, no effective implementation of Security Council resolutions could be expected."

Furthermore, major nations made it clear that their participation, and therefore the existence of the organization itself, was conditional on having veto power: "At San Francisco, the issue was made crystal clear by the leaders of the Big Five: it was either the Charter with the veto or no Charter at all" (Wilcox 1945, 954).

Even if members share common aims, they may still have vested interests and/or different views about how to best achieve these aims. In such a context, it seems hardly plausible that full, truthful, and timely communication is always possible and incentive compatible. The best way to illustrate this is perhaps to use an example. In March 1994, in the run-up to the Rwandan genocide, a number of key actors were aware of alarming pieces of intelligence (e.g., the mass training of militias, the establishment of weapon caches, and the registration of ethnic Tutsis in the capital, Kigali). Subsequent evidence shows that this intelligence was not shared with the Security Council at the time key decisions were made. ${ }^{15}$

The voting rule used by the Security Council has evolved with time, and practice, in the direction predicted by our results. Article 27(3) of

\footnotetext{
${ }_{15}$ See the report from the National Assembly of France (http:/ /www.assemblee-nationale .fr/dossiers/rwanda/r1271.asp) and the declassified documents from the National Security Archive (http://nsarchive.gwu.edu/NSAEBB/NSAEBB117/Rw01.pdf).
} 
the Charter of the United Nations makes clear that a voluntary abstention by a permanent member should be treated as a veto. But this was an early point of contention. Senator Tom Connally (US Delegate to the General Assembly of November 15, 1946) stated, "As it stands today a great power may find itself in the utterly ridiculous situation of voting for a measure which it does not entirely approve or else blocking the wheels of justice by the unwilling use of its veto. There should be some middle ground if the machinery of peaceful settlement is to function smoothly" (cited in Fassbender [1998, 182]). Such a middle ground emerged naturally as a common practice of the Security Council (Liang 1950; Stavropoulos 1967; Sievers and Daws 2014). As explained by Stavropoulos $(1967,742)$, "It has been the consistent practice of the Security Council to interpret a voluntary abstention by a permanent member as not tantamount to a veto." The direct consequence of this practice is well summarized by Fassbender (1998, 181-82): "Voluntary abstention made it possible for a permanent member to express its reservations about a particular decision while not obstructing it." 16 That such a possibility enhances information aggregation is the key mechanism behind our results.

Permanent members have repeatedly made use of the option to abstain. As a result, resolutions are frequently adopted without the explicit support of all five permanent members. ${ }^{17}$ The most striking example might be Resolution 344: “On 15 December 1973, Resolution 344 was carried by the votes of the non-permanent members, with all five permanent members abstaining" (Felsenthal and Machover 2001, 98)..$^{18}$

Consistent with our analysis, some observers of the UN seem to agree that interpreting a voluntary abstention by a permanent member as not tantamount to a veto did improve decision making in the Security Council. For instance, Liang $(1950,707)$ points out that "had the abstentions been considered as negative votes, the Security Council would have

\footnotetext{
${ }^{16}$ The Council of the European Union offers a parallel. Indeed, the Treaty of Amsterdam (1997) introduced a key novelty for matters of Common Foreign and Security Policy: instead of Unanimity, the voting procedure became unanimity rule under the constructive abstention regime. In that case, if more than a third of the member states (or member states representing more than a third of the EU population) abstain "constructively," the proposal is rejected. This rule formally corresponds to $k$-Veto, with a quorum of two-thirds of the votes.

${ }^{17}$ As tallied by Felsenthal and Machover (2001, 98), "In the period 1946-97, this happened in the case of 300 resolutions - well over $28 \%$ of the total 1068 resolutions adopted by the UNSC." Between 1998 and 2015, we counted 52 additional occurrences (voting records are available at http://www.un.org/en/sc/meetings/).

18 Also, as explained by Sievers and Daws (2014, 316-17), "Although it does not often happen, a resolution can fail to be adopted, not because of a veto, but because it does not garner sufficient affirmative votes. In contemporary practice, such instances are usually the result of a miscalculation of the voting intentions of the Council members by the sponsors of a draft resolution."
} 
adopted very few substantive decisions in its more than four years' history." Additionally, Delbruck ([1977] 1996, 302) argues that the UN Security Council "interpreted its voting rules in a way not in conformity with the respective wording of the Charter law, clearly in line, however, with the purposes and principles of the Charter, since this interpretation enabled the Security Council to act more adequately in the field of peacekeeping" (cited in Fassbender [1998, 182]).

As a matter of fact, the early reasons for, and discussion of, abstaining put forth by the permanent members can be illuminating. For instance, in 1947, the UK representative justified his abstention on Resolution 27 as follows: "The UK has abstained; but in view of the fact that everybody here clearly wishes this war to stop, the UK does not wish its abstention to be treated as a veto invalidating the resolution which has otherwise secured the necessary majority" (cited in Gross [1951, 216]).

The argument easily generalizes beyond the UN Security Council. First, the principle of national sovereignty is a pillar of international law. It implies that international organizations are limited in terms of the measures that they can effectively impose on member states. In practice, this imposes a constraint on which decision rules they can use (see, e.g., Zamora 1980; Tsebelis 2002; Sieberson 2010; Cogen 2015). ${ }^{19}$ Accordingly, Blake and Lockwood Payton (2015) find that 35 percent of the 266 intergovernmental organizations included in their database use unanimous decision making in their supreme decision-making body.

Moreover, the charters of many other international organizations leave no doubt that the promotion of common values is one of their main raisons d'être. Koremenos (2013) studies such reasons empirically and finds the resolution of uncertainty crucial. She states, "Uncertainty about the State of the World is the most common cooperation problem: two-thirds of the [intergovernmental] agreements attempt to solve it. The pervasiveness of such uncertainty is not surprising, given the numerous potential domestic and technological shocks that may affect international cooperation" (663). She also finds uncertainty about the behavior and preferences (of others) to be important.

Finally, there are many factors that hinder informal communication in international organizations (see Persico 2004). For instance, opportunity cost of time and urgency can put a limit on the time allocated to information exchange and debate. Information can also be classified or difficult to interpret by agents with different technical backgrounds. Moreover, despite the important common-value dimension, differences of views

${ }^{19}$ For instance, Posner and Sykes (2014) point out that nations are much more likely to be willing to accept nonunanimous voting systems in organizations whose decisions cannot impose high costs on its members. However, countries would also benefit from committing to ex ante beneficial agreements, although they may sometimes wind up on the losing end (see Maggi and Morelli 2006). 
or vested interests are likely to restrict truthful communication. This issue is particularly salient when agents have veto power (Austen-Smith and Feddersen 2006).

\section{B. Trials by Jury}

There is a vast literature that views trials by jury as an information aggregation problem in which the voting system plays a crucial role. The debate focuses on whether verdicts should be unanimous. The typical argument for the need for unanimous verdicts is that "it is a widely held belief among legal theorists that the requirement of unanimous jury verdicts in criminal trials reduces the likelihood of convicting an innocent defendant" (Coughlan 2000, 375). After all, according to Neilson and Winter $(2005,2)$, "the prevention of a wrongful conviction is a well-established goal of the legal system."

A rebuttal is put forth by Feddersen and Pesendorfer (1998). They show that in a game theory setup, nonunanimous verdicts protect the innocent better. This argument, which has triggered reactions and challenges (see, e.g., Coughlan 2000; Gerardi 2000; Duggan and Martinelli 2001; Persico 2004; Austen-Smith and Feddersen 2006), has not been used successfully by proponents of nonunanimous verdicts. ${ }^{20}$ Our results suggest a new angle in this debate because $k$-Veto rules combine the strengths of both Unanimity and majority rules: they foster information aggregation while still granting every juror the power to prevent a conviction.

\section{Conclusion}

In our view, in addition to their strong theoretical properties, the simplicity of $k$-Veto rules makes them particularly appealing for real-world applications. As we have discussed, there are voting bodies that use this voting system or slight variations thereof. Still, many voting bodies use unanimity or consensus, including international organizations such as the North Atlantic Treaty Organization, the Council of the European Union on most sensitive topics (excluding the Common Foreign and Security Policy), and the Southern Common Market (Mercosur). Our results suggest that (i) they should consider using a $k$-Veto rule instead, and (ii) such an institutional reform should not encounter much resistance.

\footnotetext{
${ }^{20}$ Advocates of nonunanimous verdicts may also contend that "non-unanimous verdict protects the jury from the obstinacy of the erratic or otherwise unreasonable holdout juror, decreases the likelihood of a hung jury, and reduces the costs associated with re-trying a case when the jury fails to reach a verdict" (Diamond, Rose, and Murphy 2006, 204).
} 
Any call for reform should, however, be supported by strong empirical evidence. Our companion paper, Bouton et al. (2017), is a first step in that direction. We compare Veto and Unanimity through a series of controlled laboratory experiments. By and large, we find strong support for the dominance of Veto over Unanimity.

\section{Appendix A}

\section{Equilibrium Analysis under Veto}

Proposition 1 greatly simplifies the characterization of any equilibrium under Veto since it allows us to focus on the pure common-value game in which $\mathbf{p}=\mathbf{0}$.

We organize the equilibrium analysis as follows: first, we define the pivotal events, compute their probabilities, and derive the possible actions' expected payoffs. Second, we characterize the set of equilibria. Finally, we argue that only one equilibrium is relevant.

In the common-value game, there are $n$ common-value agents, that is, $\theta_{i} \in$ $\left\{\theta_{Q}, \theta_{R}\right\}$ for all $i$. We will often refer to $\theta_{Q}$ and $\theta_{R}$ as signals instead of types.

Agents' behavior depends on pivotal events: situations in which their vote changes the final outcome toward a specific group decision. In other words, an agent is pivotal if the group decision would be different without her vote. Whether a vote is pivotal therefore depends on the decision rule and on all other agents' behavior. Agents' behavior, in turn, depends on their strategies and on the signal they receive. Thus, for any strategy profile, it is possible to compute the probability of each pivotal event.

At this point, it is useful to introduce two new objects. First, $x_{a}$ denotes, from the perspective of a given agent, the number of other agents playing action $a$. Second, $\tau_{a}^{\omega}(\sigma)$ denotes the state-contingent probability that an agent votes $a$ in state $\omega$ for a given strategy profile $\sigma$. It is defined as follows:

$$
\tau_{a}^{\omega}(\sigma) \equiv \sum_{\theta \in\left\{\theta_{Q}, \theta_{R}\right\}} \sigma_{a}(\theta) \operatorname{Pr}(\theta \mid \omega),
$$

where $\operatorname{Pr}\left(\theta_{R} \mid \omega\right)=\operatorname{Pr}\left(s_{R} \mid \omega\right)$ and $\operatorname{Pr}\left(\theta_{Q} \mid \omega\right)=\operatorname{Pr}\left(s_{Q} \mid \omega\right)$ for all $\omega$ since $\mathbf{p}=\mathbf{0}$.

\section{Pivot Probabilities and Payoffs}

Under Veto, there are two pivotal events. First, an $r$-vote is pivotal when, without that vote, $R$ is lacking just one vote to be adopted (i.e., $x_{r}=(n-1) / 2$ and nobody casts a $v$-vote). We denote that pivotal event in state $\omega$ by $\operatorname{piv}_{R}^{\omega}$. Second, a $v$-vote is pivotal when the number of $r$-votes among other agents is larger than or equal to the quorum (i.e., $x_{r} \geq(n+1) / 2$ ) and nobody else casts a $v$-vote. We denote that pivotal event in state $\omega$ by piv ${ }_{Q}^{\omega}$. Importantly, a $q$-ballot is never pivotal under Veto. Indeed, this would require that, without that vote, $R$ wins (i.e., $x_{r} \geq(n+1) / 2$ ), and, with that vote, $Q$ wins (i.e., $\left.x_{r}<(n+1) / 2\right)$, an impossibility.

For the sake of readability, our notation does not reflect the fact that the probability of pivotal events depends on the strategies through the expected vote shares; that is, we henceforth omit $\sigma$ from the notation. 
For piv ${ }_{R}^{\omega}$, we have

$$
\operatorname{Pr}\left(\operatorname{piv}_{R}^{\omega}\right)=\left(\begin{array}{c}
n-1 \\
\frac{n-1}{2}
\end{array}\right)\left(\tau_{q}^{\omega}\right)^{(n-1) / 2}\left(\tau_{r}^{\omega}\right)^{(n-1) / 2}
$$

Similarly, for piv $_{Q}^{\omega}$ we have

$$
\operatorname{Pr}\left(\operatorname{piv}_{Q}^{\omega}\right)=\sum_{j=(n+1) / 2}^{n-1}\left(\begin{array}{c}
n-1 \\
j
\end{array}\right)\left(\tau_{r}^{\omega}\right)^{j}\left(\tau_{q}^{\omega}\right)^{n-1-j} .
$$

Using these pivot probabilities, we can compute the expected payoff of the different actions for a common-value agent of type $\theta \in\left\{\theta_{Q}, \theta_{R}\right\}$. To do this, it is useful to define common-value agents' interim beliefs about the state of nature:

$$
\begin{aligned}
& \operatorname{Pr}\left(\omega_{Q} \mid \theta\right)=\frac{\operatorname{Pr}\left(\theta \mid \omega_{Q}\right)}{\operatorname{Pr}\left(\theta \mid \omega_{Q}\right)+\operatorname{Pr}\left(\theta \mid \omega_{R}\right)}, \\
& \operatorname{Pr}\left(\omega_{R} \mid \theta\right)=\frac{\operatorname{Pr}\left(\theta \mid \omega_{R}\right)}{\operatorname{Pr}\left(\theta \mid \omega_{R}\right)+\operatorname{Pr}\left(\theta \mid \omega_{Q}\right)} .
\end{aligned}
$$

Therefore, we have that the expected payoff of an $r$-vote for a common-value agent who received signal $\theta$ is

$$
G(r \mid \theta)=\operatorname{Pr}\left(\omega_{R} \mid \theta\right) \operatorname{Pr}\left(\operatorname{piv}_{R}^{\omega_{R}}\right)-\operatorname{Pr}\left(\omega_{Q} \mid \theta\right) \operatorname{Pr}\left(\operatorname{piv}_{R}^{\omega_{Q}}\right),
$$

the expected payoff of a $v$-vote for a common-value agent who received signal $\theta$ is

$$
G(v \mid \theta)=\operatorname{Pr}\left(\omega_{Q} \mid \theta\right) \operatorname{Pr}\left(\operatorname{piv}_{Q}^{\omega_{Q}}\right)-\operatorname{Pr}\left(\omega_{R} \mid \theta\right) \operatorname{Pr}\left(\operatorname{piv}_{Q}^{\omega_{R}}\right),
$$

and the expected payoff of a $q$-vote for a common-value agent who received signal $\theta$ is

$$
G(q \mid \theta)=0
$$

\section{The Information-Aggregation Equilibrium}

To organize the discussion of the equilibrium behavior of common-value agents under Veto, it is useful to partition the parameter space. The reason is that equilibrium strategies are nontrivially affected by the relative precision of the signals. We denote the precision of a signal $\theta_{Q}$ by $\phi_{Q} \equiv \operatorname{Pr}\left(\theta_{Q} \mid \omega_{Q}\right) / \operatorname{Pr}\left(\theta_{Q} \mid \omega_{R}\right) \in(1, \infty)$ and that of signal $\theta_{R}$ by $\phi_{R} \equiv \operatorname{Pr}\left(\theta_{R} \mid \omega_{R}\right) / \operatorname{Pr}\left(\theta_{R} \mid \omega_{Q}\right) \in(1, \infty) .{ }^{21}$ For any $n$ and $\phi_{R}$, we have identified four thresholds $\phi_{1}, \phi_{2}, \phi_{3}$, and $\phi_{4}$ for $\phi_{Q}$, at which the set of actions played with strictly positive probability in equilibrium changes. The next

${ }^{21}$ Conversely, we have

$$
\operatorname{Pr}\left(\theta_{Q} \mid \omega_{Q}\right)=\frac{\left(1-\phi_{R}\right) \phi_{Q}}{1-\phi_{Q} \phi_{R}}
$$

and

$$
\operatorname{Pr}\left(\theta_{Q} \mid \omega_{R}\right)=\frac{1-\phi_{R}}{1-\phi_{Q} \phi_{R}}
$$


proposition characterizes these thresholds and the equilibrium associated with it.

Proposition 6 (Information-aggregation equilibrium). For each tuple $\left(\phi_{R}\right.$, $\left.\phi_{Q}, n\right)$ there exist unique thresholds $\phi_{1}, \phi_{2}, \phi_{3}$, and $\phi_{4}$ (with $1<\phi_{1}<\phi_{2} \leq \phi_{3} \leq$ $\left.\phi_{4}<\infty\right)$ such that the following strategy profile is a responsive symmetric equilibrium under Veto:

$$
\begin{gathered}
\sigma_{r}\left(\theta_{R}\right)=1-\epsilon^{*}-\gamma^{*}, \quad \sigma_{q}\left(\theta_{R}\right)=\epsilon^{*}+\gamma^{*}, \quad \sigma_{v}\left(\theta_{R}\right)=0, \\
\sigma_{r}\left(\theta_{Q}\right)=\eta^{*}, \quad \sigma_{q}\left(\theta_{Q}\right)=1-\eta^{*}-\delta^{*}, \quad \sigma_{v}\left(\theta_{Q}\right)=\delta^{*},
\end{gathered}
$$

where $\eta^{*}, \epsilon^{*}, \gamma^{*}$, and $\delta^{*}$ are unique and satisfy the following properties:

\begin{tabular}{cccccc}
\hline \hline & $\phi_{Q}<\phi_{1}$ & $\phi_{Q} \in\left[\phi_{1}, \phi_{2}\right]$ & $\phi_{Q} \in\left(\phi_{2}, \phi_{3}\right]$ & $\phi_{Q} \in\left(\phi_{3}, \phi_{4}\right)$ & $\phi_{Q} \geq \phi_{4}$ \\
\hline$\eta^{*}$ & {$[0,1)$} & 0 & 0 & 0 & 0 \\
$\epsilon^{*}$ & 0 & 0 & $(0,1)$ & 0 & 0 \\
$\gamma^{*}$ & 0 & 0 & 0 & $\left(0,1-\delta^{*}\right)$ & 0 \\
$\delta^{*}$ & 0 & 0 & 0 & $(0,1)$ & 1 \\
\hline
\end{tabular}

Proof. See the online appendix.

Figure A1 illustrates proposition 6 in the space $\left(\phi_{R}, \phi_{Q}\right)$ for $n=7$. In short, the voting behavior under Veto is as follows. For $\phi_{Q} \in\left[1, \phi_{3}\right]$, the behavior of commonvalue agents under Veto is the same as it would be under majority rule, and for $\phi_{Q} \in\left[\phi_{4}, \infty\right)$, it is the same as it would be under Unanimity. For $\phi_{Q} \in\left(\phi_{3}, \phi_{4}\right)$, all actions are played with positive probability. Therefore, the behavior under Veto is necessarily different than under majority rule or Unanimity. We now detail the mechanisms behind the behavior of common-value agents for the different values of $\phi_{Q}$.

When $\phi_{Q} \in\left[\phi_{1}, \phi_{2}\right]$, the precision of the two signals is not too different. The equilibrium is then such that $\sigma_{r}\left(\theta_{R}\right)=1=\sigma_{q}\left(\theta_{Q}\right)$ : common-value agents vote their signal and do not use their veto. The intuition is the following. An $r$-ballot is pivotal if there are $(n-1) / 2$ votes for $R$ in the group of other agents. Given the strategy under consideration, this requires that in the group of other agents, there are $(n-1) / 2$ signals $\theta_{Q}$ and $(n-1) / 2$ signals $\theta_{R}$. Adding one's own signal to this count means a lead of one signal in favor of one of the two states. The condition $\phi_{Q} \in\left[\phi_{1}, \phi_{2}\right]$ ensures that, in such a case, the posterior beliefs of the agent under consideration are in line with her signal. In other words, conditional on an $r$-ballot being pivotal, $\theta_{R}$-agents believe that $R$ is best and $\theta_{Q}$-agents believe that $Q$ is best. The implication in terms of voting behavior is obvious for $\theta_{R}$-agents: they vote for $r$. For $\theta_{Q}$-agents, the situation is slightly more complicated since there are two options to vote against $R$ : voting either $q$ or $v$. A $q$-ballot has an expected payoff of zero, whereas a $v$-ballot has a negative expected payoff. This is so because a $v$-ballot changes the outcome when there are $(n+1) / 2$ or more $r$ votes. Given the strategy under consideration and the relative precision of the signals, this is more likely to happen in state $\omega_{R}$ than in state $\omega_{Q}$.

When $\phi_{Q} \in\left[1, \phi_{1}\right)$, signal $\theta_{R}$ is more precise than signal $\theta_{Q}$. In that case, $\theta_{Q^{-}}$ agents prefer to overlook their signal and vote $r$ with positive probability. Doing so, they "compensate" for the bias in the information structure. The reason is 


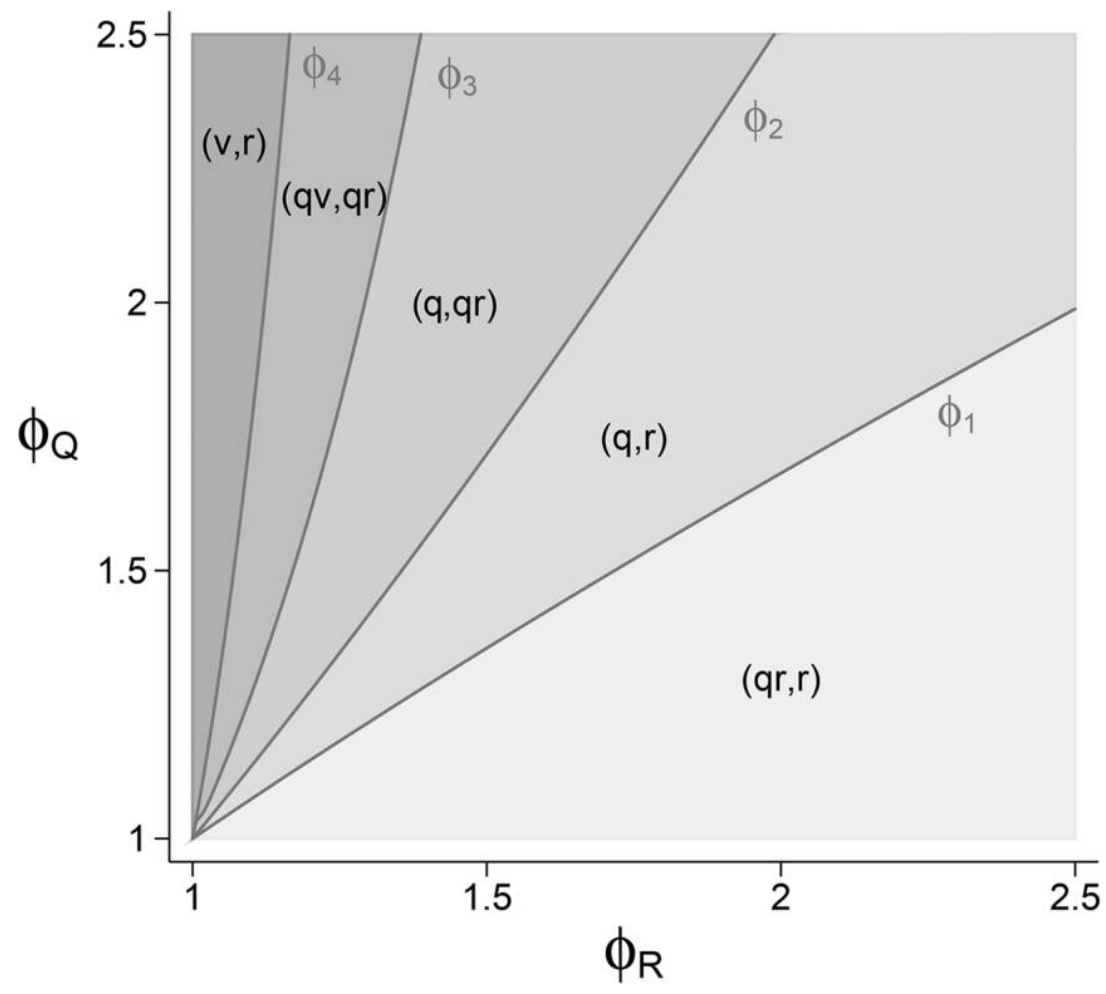

FIG. A1.-Equilibrium strategies under Veto for $n=7$. The letters in parentheses refer to the strategy of the common-value agents. The first element of each couple refers to the strategy of $\theta_{Q}$-agents. The second element of each couple refers to the strategy of $\theta_{R}$-agents. Pure strategies are denoted by single letters; mixed strategies are denoted by coupled letters.

that, because the signal is imprecise, the probability of making a mistake in state $\omega_{R}$ is too high. This is exactly the same behavior as under majority rule.

The case with $\phi_{Q} \in\left(\phi_{2}, \phi_{3}\right]$ resembles the one with $\phi_{Q} \in\left[1, \phi_{1}\right)$, but the difference in signal precision is in favor of $\theta_{Q}$. As a result of the difference in signal precision, $\theta_{R}$-agents prefer to overlook their signal and vote against $R$ with positive probability. Again, this is similar to the case under majority rule. Yet, under Veto agents have two ways to vote against $R$ : voting $q$ or $v$. The appeal of $v$ depends positively on the precision of the $\theta_{Q}$ signal and negatively on the relative probability of being pivotal in favor of $Q$ in states $\omega_{R}$ and $\omega_{Q}$. Therefore, $\phi_{3}$ requires that, for a given precision of the $\theta_{Q}$ signal, the expected lead of $R$ in state $\omega_{R}$ is large enough and/or the lead of $Q$ in state $\omega_{Q}$ small enough.

When $\phi_{Q} \in\left(\phi_{3}, \phi_{4}\right)$, the situation resembles the situation for $\phi_{Q} \in\left(\phi_{2}, \phi_{3}\right]$. The difference is that $\theta_{Q}$-agents want to use their veto power with positive probability. As just explained, this is so because, for the strategy profile when $\phi_{Q} \in\left(\phi_{2}, \phi_{3}\right]$, the expected lead of $R$ in state $\omega_{R}$ is too large in comparison to the expected lead 
of $Q$ in state $\omega_{Q}$. In equilibrium, (1) $\theta_{R}$-agents mix between $r$ and $q$, but they vote $r$ with higher probability than for $\phi_{Q} \leq \phi_{3}$; and (2) $\theta_{Q}$-agents mix between $q$ and $v$. The intuition comes in two steps. First, the positive probability of a veto by

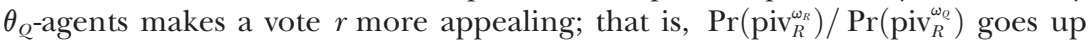
since there are more $\theta_{Q}$-agents in state $\omega_{Q}$. Second, the relatively higher vote shares of $R$ in state $\omega_{R}$ make a $v$-vote less appealing; that is, $\operatorname{Pr}\left(\operatorname{piv}_{Q}^{\omega_{R}}\right)$ goes up and $\operatorname{Pr}\left(\operatorname{piv}_{Q}^{\omega_{Q}}\right)$ goes down.

When $\phi_{Q} \in\left[\phi_{4}, \infty\right)$, common-value agents behave as they would under Unanimity. The $\theta_{Q}$ signal is so precise (relatively) that one $\theta_{Q}$ signal is sufficient information to conclude that $Q$ is better than $R$ (even if all other signals are $\theta_{R}$ signals). Therefore, $\theta_{Q}$-agents prefer to cast a $v$-vote. For $\theta_{R}$-agents, the situation is different. Given the strategy under consideration, conditional on being pivotal, all other agents must have received a $\theta_{R}$-signal. Obviously, if there are only $\theta_{R}$ signals, any agent must believe that state $\omega_{R}$ is more likely than state $\omega_{Q}$. She thus prefers to cast an $r$-vote.

\section{The Unanimity-Like Equilibrium}

We show here that, when $\phi_{Q}<\phi_{4}$, there exists another symmetric responsive equilibrium that corresponds to the unique equilibrium under Unanimity (in the next subsection, we show that this is the only other equilibrium). However, such an equilibrium is not robust under Veto (see discussion below). We therefore see it as of little relevance.

Proposition 7 (Unanimity-like equilibrium). If $\phi_{Q}<\phi_{4}$, the following strategy profile is a responsive symmetric equilibrium under Veto:

$$
\begin{aligned}
& \sigma_{r}\left(\theta_{R}\right)=1, \quad \sigma_{q}\left(\theta_{R}\right)=0, \quad \sigma_{v}\left(\theta_{R}\right)=0, \\
& \sigma_{r}\left(\theta_{Q}\right)=\alpha^{*}, \quad \sigma_{q}\left(\theta_{Q}\right)=0, \sigma_{v}\left(\theta_{Q}\right)=1-\alpha^{*},
\end{aligned}
$$

where

$$
\alpha^{*}=\frac{\left(\phi_{Q}-1\right)\left[\phi_{R}-\left(\phi_{Q}\right)^{1 /(n-1)}\right]}{\left(\phi_{R}-1\right)\left[\left(\phi_{Q}\right)^{1 /(n-1)} \phi_{Q}-1\right]} \in[0,1) .
$$

This equilibrium corresponds to the unique equilibrium under Unanimity.

Proof. Straightforward extension of Feddersen and Pesendorfer (1998). See appendix B for detail.

Under Unanimity, $\theta_{Q}$-agents realize they can be pivotal only if all other agents vote $r$. Given that this is more likely to happen in state $\omega_{R}$ (because $\theta_{R}$-agents always play $r$ ), $\theta_{Q}$-agents play $v$ with probability one only if signal $s_{Q}$ is sufficiently precise $\left(\phi_{Q} \geq \phi_{4}\right)$. In all other cases, they play $r$ with positive probability, which results in a relatively high probability of errors of both types (Feddersen and Pesendorfer 1998).

To understand why it is also an equilibrium under Veto, remember that we can redefine Unanimity using the same decision rule as under Veto. Then Unanimity corresponds to Veto with a smaller action set. That is, action $q$ is not available under Unanimity. Under Veto, when no other agent ever votes $q$, a $q$-vote is strategically equivalent to an $r$-vote (since the approval quorum is satisfied with prob- 
ability one, the reform will be adopted unless someone vetoes it). ${ }^{22}$ So the equilibrium under Unanimity must be an equilibrium under Veto.

However, we see several reasons to question the robustness of such an equilibrium under Veto. First, as we show in the welfare analysis, it is Pareto dominated by the information-aggregation equilibrium.

Second, it is instable in the following sense. Imagine that $\theta_{Q}$-agents tremble and play $q$ with very small but strictly positive probability $\xi$ (in equilibrium, they are indifferent between the three possible actions), while $\theta_{R}$-agents still play $r$ with probability one. Then a best response for $\theta_{Q}$-agents cannot involve playing both $r$ and $v$ with strictly positive probability; that is, it cannot be "close" to the equilibrium strategy profile. In fact, as $\xi$ tends to zero, the equilibrium of such a perturbed game tends to the information-aggregation equilibrium. The intuition is the following: in the unanimity-like equilibrium, agents are indifferent between $q$ and $r$ because one can never be pivotal in favor of $R$ (if no other agent has vetoed, then it must be that everyone else played $r$, and there already is a majority in favor of the reform). But if $q$ is played with positive probability, even very small, it becomes possible that $x_{r}=(n-1) / 2$. Since this is more likely to happen in state $Q$ than in state $R$, a $\theta_{Q}$-agent then strictly prefers to vote $q$ than $r$. Note that if $\theta_{Q^{-}}$-agents do not play $r$, this decreases the gain for them to play $v$, and the equilibrium unravels. ${ }^{23}$

Finally, we present in a companion paper the results of an experimental study that strongly supports the prediction that agents will coordinate on the informationaggregation equilibrium rather than the unanimity-like equilibrium (see Bouton et al. 2017).

\section{No Other Equilibria}

We show that there cannot be other responsive symmetric equilibria than those described in propositions 6 and 7. The proof is rather straightforward but quite tedious, so we organize it with the matrix in figure A2 that considers all the possible classes of (symmetric) strategy profiles. Possible classes of strategy for an agent are given by $\{r, r q, q, q v, v, q r v, r v\}$, where for instance $r q$ means that this agent plays $r$ and $q$ (but not $v$ ) with strictly positive probability.

We show in five steps that the only possible equilibria correspond to the information-aggregation equilibrium (cells "IAE") and the unanimity-like one (cells "ULE”).

First, note that if agent $\theta_{Q}$ plays $r$ with positive probability at equilibrium, it must be the case that agent $\theta_{R}$ plays $r$ with probability one because signals are informative. Formally, from equations (A3), (A4), and (A5), we have

$$
G\left(r \mid \theta_{Q}\right) \geq 0 \Rightarrow G\left(r \mid \theta_{R}\right)>0
$$

and

${ }^{22}$ In terms of information aggregation, playing $q$ at such an equilibrium could convey information, but such information would not be exploited in making the group decision.

${ }^{23}$ The equilibrium is, however, trembling-hand perfect because it is possible to find a joint sequence of tremble for all agents that tends to zero and a corresponding sequence of equilibria that converges toward the equilibrium. However, it is easy to show that such sequences must have the unappealing feature that trembles make agents $\theta_{R}$ more likely to vote for $q$ than agents $\theta_{Q}$. 


\begin{tabular}{|c|c|c|c|c|c|c|c|}
\hline & \multicolumn{7}{|c|}{$\theta_{R}$-Agents } \\
\hline & $r$ & $r q$ & $q$ & qv & rqv & rv & $\mathbf{v}$ \\
\hline \multirow{6}{*}{ 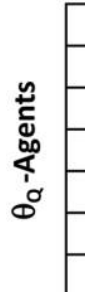 } & & $x$ & $x$ & $x$ & $x$ & $x$ & $\mathrm{x}$ \\
\hline & IAE & $\mathrm{x}$ & $x$ & $x$ & $x$ & $x$ & $\mathrm{x}$ \\
\hline & $\mathrm{IAE}$ & IAE & & $x$ & $x$ & $x$ & $\mathrm{x}$ \\
\hline & no veto & IAE & & $x$ & $x$ & $x$ & $\mathrm{x}$ \\
\hline & no veto & $x$ & $x$ & $x$ & $x$ & $x$ & $\mathrm{x}$ \\
\hline & ULE & $x$ & $x$ & $x$ & $x$ & $x$ & $\mathrm{x}$ \\
\hline v & IAE & Prop 6 & & & Prop 6 & Prop 6 & \\
\hline
\end{tabular}

FIG. A2.-All possible classes of (symmetric) strategy profiles. For instance, strategy rq for agents of type $z$ means that they play $r$ and $q$ (but not $v$ ) with strictly positive probability.

$$
G\left(r \mid \theta_{Q}\right) \geq G\left(v \mid \theta_{Q}\right) \Rightarrow G\left(r \mid \theta_{R}\right)>G\left(v \mid \theta_{R}\right) .
$$

Similarly, if agent $\theta_{R}$ plays $v$ with positive probability, then agent $\theta_{Q}$ plays $v$ with probability one. These two restrictions rule out the cases corresponding to the shaded cells with reference "x."

Second, we can rule out a series of remaining cases in which the strategy profile is not responsive. These are the cells in dark gray.

Third, from the characterization of the information-aggregation equilibrium, we have that (i) if agents $\theta_{Q}$ play $v$ with probability one, then agents $\theta_{R}$ can be pivotal only if all agents receive a signal $\theta_{R}$, in which case they strictly prefer to play $r$. We can therefore rule out another set of profiles. The corresponding cells are shaded and labeled "Prop 6."

Fourth, from equation (A5), it is easy to show that, if agents $\theta_{Q}$ play $q v$ or $q r v$, then $\theta_{R}$-agents play $r$ with probability one only if $\phi_{Q} \leq \phi_{2}$. But then, we have that $G\left(v \mid \theta_{Q}\right)<0$, a contradiction. We can therefore rule out these profiles as well. The corresponding cells are shaded and labeled "no veto."

Finally, the remaining cells correspond to the two equilibria we have characterized. And we have shown that they are both unique within their strategy profile class.

\section{Appendix B}

\section{Equilibrium Analysis under Unanimity}

In this appendix, we characterize the unique responsive equilibrium under Unanimity. Doing so, we extend the equilibrium characterization in Feddersen and Pesendorfer (1998) to biased information structure and the possible presence of a private-value agent.

As under Veto, $\theta_{P}$-agents strictly prefer to play $v$, that is, $\sigma_{v}^{U}\left(\theta_{P}\right)=1$, in any responsive symmetric equilibrium under Unanimity. It is therefore straightforward to extend proposition 1.

Proposition 8. Under Unanimity, for any vector $\mathbf{p}, \sigma^{*}$ is an equilibrium under Unanimity if and only if

i. private-value agents veto the reform,

ii. $\sigma^{*}$ is an equilibrium of the corresponding game with $\mathbf{p}=\mathbf{0}$. 
Proof. Identical to that of proposition 1.

As for Veto, this proposition greatly simplifies the equilibrium analysis by allowing us to focus on the pure common-value game in which $\mathbf{p}=\mathbf{0}$. This is exactly what we do in the remainder of this appendix.

\section{Pivot Probabilities and Expected Payoffs}

Under Unanimity, a vote for $r$ is pivotal if and only if, without that vote, the group decision is $Q$ but, with that vote, it becomes $R$. This happens when no other agent is casting a $v$-vote (i.e., $x_{v}=0$ ). In this case, we say that the vote is pivotal in favor of $R$. We denote this event in state $\omega$ by $\operatorname{piv}_{R}^{\omega, U}$. The probability of that event depends on expected vote shares, which in turn depend on the state of the world and agent strategies. We denote $\operatorname{Pr}\left(\operatorname{piv}_{R}^{\omega, U}\right)$ the probability of being pivotal in favor of $R$ in state $\omega$ under Unanimity. Formally,

$$
\operatorname{Pr}\left(\operatorname{piv}_{R}^{\omega, U}\right)=\left(\tau_{r}^{\omega}\right)^{n-1}
$$

A vote for $v$ cannot be pivotal. Indeed, there is no combination of other agents' vote such that the decision is $R$ without this vote and becomes $Q$ with it. In fact, either $x_{v}>0$ and the "current" outcome, $Q$, can no longer be changed or $x_{v}=0$, which implies $x_{r}=n-1<n$, and the "current" outcome is $Q$. Remember that $q$ is not an available action under $U$.

The expected payoff of actions $r$ for an agent of type $\theta \in\left\{\theta_{R}, \theta_{Q}\right\}$ under Unanimity is

$$
G^{U}(r \mid \theta)=\operatorname{Pr}\left(\omega_{R} \mid \theta\right) \operatorname{Pr}\left(\operatorname{piv}_{R}^{\omega_{R}, U}\right)-\operatorname{Pr}\left(\omega_{Q} \mid \theta\right) \operatorname{Pr}\left(\operatorname{piv}_{R}^{\omega_{Q}, U}\right),
$$

and the expected payoff of actions $v$ for an agent with signal $\theta \in\left\{\theta_{R}, \theta_{Q}\right\}$ under Unanimity is

$$
G^{U}(v \mid \theta)=0
$$

\section{The Unique Equilibrium}

The following proposition extends the equilibrium characterization in Feddersen and Pesendorfer (1998) to possibly biased information structures and the possible presence of private-value agents.

Proposition 9. The following strategy profile is the unique responsive symmetric equilibrium under Unanimity:

i. If $\phi_{Q}<\phi_{4}$, then

$$
\begin{aligned}
& \sigma_{r}^{* U}\left(\theta_{R}\right)=1, \\
& \sigma_{r}^{* U}\left(\theta_{Q}\right)=\alpha^{*}, \\
& \sigma_{v}^{* U}\left(\theta_{Q}\right)=1-\alpha^{*},
\end{aligned}
$$


with

$$
\alpha^{*}=\frac{\left(\phi_{Q}-1\right)\left[\phi_{R}-\left(\phi_{Q}\right)^{1 /(n-1)}\right]}{\left(\phi_{R}-1\right)\left[\left(\phi_{Q}\right)^{1 /(n-1)} \phi_{Q}-1\right]} \in(0,1) .
$$

ii. If $\phi_{Q} \geq \phi_{4}$, then

$$
\sigma_{r}^{* U}\left(\theta_{R}\right)=1 \text { and } \sigma_{v}^{* U}\left(\theta_{Q}\right)=1 .
$$

Proof. See the online appendix.

The intuition is easier to grasp by first explaining why and when $\theta_{R^{-}}$-agents voting $r$ and $\theta_{Q}$-agents voting $v$ is not an equilibrium (i.e., $\phi_{Q}<\phi_{4}$ ). Consider an agent who receives signal $\theta_{Q}$. She believes, but is not sure, that $Q$ is a better decision than $R$. When deciding which vote to cast, she focuses only on situations in which her vote is pivotal. Under Unanimity, this happens only when all other agents vote for $r$ (event pivive $v_{R}^{\omega, U}$ ). If $\theta_{R}$-agents vote $r$ and $\theta_{Q}$-agents vote $v$, this happens if all other agents have received a signal $\theta_{R}$, which is more likely to happen in state $\omega_{R}$ than in state $\omega_{Q}$. As long as the precision of the $\theta_{Q}$ signal is not too high (i.e., $\left.\phi_{Q}<\phi_{4}\right)$, the joint event $\left(n-1 \theta_{R}\right.$-signals and one $\theta_{Q}$-signal) is also more likely in state $\omega_{R}$ than in state $\omega_{Q}$. She is thus better overlooking her signal and voting for $r$. Therefore, $\theta_{R}$-agents voting $r$ and $\theta_{Q^{-}}$-agents voting $v$ cannot be an equilibrium in this case.

To understand why the equilibrium is in mixed strategies when $\phi_{Q}<\phi_{4}$, notice that when $\theta_{Q}$-agents mix between $r$ and $v$, the information content conditional on being pivotal decreases (since $\theta_{Q}$-agents also vote $r$ with positive probability, the fact of being pivotal no longer hinges on all other agents having received $\theta_{R}$-signals; hence, the posterior probability of being in state $\omega_{R}$ when being pivotal decreases). For $\sigma_{r}^{U}\left(\theta_{Q}\right)$ large enough, this information content is too low to convince $\theta_{Q}$-agents to overlook their signal. The equilibrium corresponds to the case in which the posterior probability of being in either state is equal, which makes agents indifferent between the two actions.

For $\phi_{Q} \geq \phi_{4}$, the precision of the $\theta_{Q}$-signal is so high that a $\theta_{Q}$-agent remains convinced that state $\omega_{Q}$ is more likely when all other agents received a $\theta_{R}$ signal. In other words, a single $\theta_{Q}$-signal would suffice to convince an agent that could observe the $n$ signals and could decide for the group to choose decision $Q$. Therefore, $\theta_{R}$-agents voting $r$ and $\theta_{Q}$-agents voting $v$ is an equilibrium. Note that there always is an $n$ sufficiently large that this case does not arise.

\section{Appendix C}

\section{Proofs}

\section{Proof of Lemma 1}

Given proposition 1, we can focus on $\mathbf{p}=\mathbf{0}$.

First, note that $v$ is a weakly dominant strategy for $\theta_{Q}$-agents when $\phi_{Q}>\left(\phi_{R}\right)^{n-1}$. The proof that $\theta_{R}$-agents play $r$ under $k$-Veto is similar to step $\mathrm{v}$ of the proof of proposition 6 (the proposition is in app. A and the proof in the online appendix). 
Second, we prove that, for each tuple $\left(\phi_{Q}, \phi_{R}, n, \mathbf{p}\right), \theta_{R}$-agents playing $r$ and $\theta_{Q}$-agents playing $q$ is an equilibrium if

$$
\phi_{Q} \in\left[\left(\phi_{R}\right)^{(k-1) /(n+1-k)},\left(\phi_{R}\right)^{k /(n-k)}\right) .
$$

To do so, we first need to define the gains to vote $r$ or $v$ for each common-value agent type under $k$-Veto when $\theta_{R}$-agents play $r$ and $\theta_{Q}$-agents play $q$ :

$$
\begin{aligned}
& G^{k}\left(r \mid \theta_{R}\right) \equiv \frac{\phi_{R}}{\phi_{R}+1}\left(\begin{array}{c}
n-1 \\
\frac{n-1}{2}
\end{array}\right)\left[\operatorname{Pr}\left(\theta_{R} \mid \omega_{R}\right)\right]^{k-1}\left[\operatorname{Pr}\left(\theta_{Q} \mid \omega_{R}\right)\right]^{n-k} \\
& -\frac{1}{\phi_{R}+1}\left(\begin{array}{c}
n-1 \\
\frac{n-1}{2}
\end{array}\right)\left[\operatorname{Pr}\left[\theta_{R} \mid \omega_{Q}\right)\right]^{k-1}\left[\operatorname{Pr}\left(\theta_{Q} \mid \omega_{Q}\right)\right]^{n-k}, \\
& G^{k}\left(r \mid \theta_{Q}\right) \equiv \frac{1}{\phi_{Q}+1}\left(\begin{array}{c}
n-1 \\
\frac{n-1}{2}
\end{array}\right)\left[\operatorname{Pr}\left(\theta_{R} \mid \omega_{R}\right)\right]^{k-1}\left[\operatorname{Pr}\left(\theta_{Q} \mid \omega_{R}\right)\right]^{n-k} \\
& -\frac{\phi_{Q}}{\phi_{Q}+1}\left(\begin{array}{c}
n-1 \\
\frac{n-1}{2}
\end{array}\right)\left[\operatorname{Pr}\left(\theta_{R} \mid \omega_{Q}\right)\right]^{k-1}\left[\operatorname{Pr}\left(\theta_{Q} \mid \omega_{Q}\right)\right]^{n-k}, \\
& G^{k}\left(v \mid \theta_{R}\right) \equiv \frac{1}{\phi_{R}+1} \sum_{j=k}^{n-1}\left(\begin{array}{c}
n-1 \\
j
\end{array}\right)\left[\operatorname{Pr}\left(\theta_{R} \mid \omega_{Q}\right)\right]^{j}\left[\operatorname{Pr}\left(\theta_{Q} \mid \omega_{Q}\right)\right]^{n-1-j} \\
& -\frac{\phi_{R}}{\phi_{R}+1} \sum_{j=k}^{n-1}\left(\begin{array}{c}
n-1 \\
j
\end{array}\right)\left[\operatorname{Pr}\left(\theta_{R} \mid \omega_{R}\right)\right]^{j}\left[\operatorname{Pr}\left(\theta_{Q} \mid \omega_{R}\right)\right]^{n-1-j}, \\
& G^{k}\left(v \mid \theta_{Q}\right) \equiv \frac{\phi_{Q}}{\phi_{Q}+1} \sum_{j=k}^{n-1}\left(\begin{array}{c}
n-1 \\
j
\end{array}\right)\left[\operatorname{Pr}\left(\theta_{R} \mid \omega_{Q}\right)\right]^{j}\left[\operatorname{Pr}\left(\theta_{Q} \mid \omega_{R}\right)\right]^{n-1-j} \\
& -\frac{1}{\phi_{Q}+1} \sum_{j=k}^{n-1}\left(\begin{array}{c}
n-1 \\
j
\end{array}\right)\left[\operatorname{Pr}\left(\theta_{R} \mid \omega_{R}\right)\right]^{j}\left[\operatorname{Pr}\left(\theta_{Q} \mid \omega_{R}\right)\right]^{n-1-j} .
\end{aligned}
$$

For $\sigma_{r}\left(\theta_{R}\right)=1$ and $\sigma_{q}\left(\theta_{Q}\right)=1$ to be an equilibrium, we need $(a) G^{k}\left(r \mid \theta_{R}\right) \geq 0$, (b) $G^{k}\left(r \mid \theta_{Q}\right) \leq 0,(c) G^{k}\left(v \mid \theta_{Q}\right) \leq 0$, and $(d) G^{k}\left(r \mid \theta_{R}\right) \geq G^{k}\left(v \mid \theta_{R}\right)$. From $G^{k}\left(v \mid \theta_{Q}\right) \geq$ $G^{k}\left(v \mid \theta_{R}\right)$ and conditions $a$ and $c$, we have that $d$ is necessarily satisfied. It remains to prove that conditions $a, b$, and $c$ are satisfied.

Consider condition $a$. In this case, $G^{k}\left(r \mid \theta_{R}\right) \geq 0$ boils down to

$$
\phi_{R} \geq\left(\frac{1}{\phi_{R}}\right)^{k-1}\left(\phi_{Q}\right)^{n-k}
$$

which is satisfied iff $\phi_{Q} \leq\left(\phi_{R}\right)^{k /(n-k)}$, which holds by assumption. 
Consider condition $c$. In this case, $G^{k}\left(r \mid \theta_{Q}\right) \leq 0$ boils down to

$$
\frac{1}{\phi_{Q}} \leq\left(\frac{1}{\phi_{R}}\right)^{k-1}\left(\phi_{Q}\right)^{n-k},
$$

which is satisfied iff $\left(\phi_{R}\right)^{(k-1) /(n+1-k)} \leq \phi_{Q}$, which holds by assumption.

Consider condition $d$. Following an approach identical to that in the proof of proposition 6 (for the equivalent condition), we obtain a term-by-term sufficient condition for $G^{k}\left(v \mid \theta_{Q}\right) \leq 0$ :

$$
\phi_{Q} \leq\left(\phi_{R}\right)^{j /(n-j)} .
$$

From $\phi_{Q} \leq\left(\phi_{R}\right)^{k /(n-k)}, \phi_{R}>1$, and

$$
\frac{j}{n-j} \geq \frac{k}{n-k} \quad \forall j \in\{k, \ldots, n-1\},
$$

we have that this is satisfied. QED

\section{Proof of Theorem 1}

To prove the theorem, we show that all agents are weakly better off under Veto at the information-aggregation equilibrium (we denote the corresponding mechanism by $\left.V_{\sigma^{u}}\right)$ than at the unique equilibrium under Unanimity $\left(U_{\sigma^{v}}\right)$ and that, unless $\theta_{i}=\theta_{P}$ or $\phi_{Q} \geq \phi_{4}$, some agents are strictly better off.

First, $V_{\sigma^{u}}(\boldsymbol{\theta})=U_{\sigma^{v}}(\boldsymbol{\theta})=Q$ when there exists $i$ such that $\theta_{i}=\theta_{P}$ or $\phi_{Q} \geq \phi_{4}$. In these cases, all agents are equally well off ex post and, therefore, at the interim stage.

Now consider $\theta_{i} \neq \theta_{P}$ for all $i$ and $\phi_{Q}<\phi_{4}$. The game is equivalent to the pure common-value game (i.e., $\mathbf{p}=\mathbf{0}$ ). We show that

$$
u_{i}\left(V_{\sigma^{n}} \mid \theta_{i}\right)>u_{i}\left(U_{\sigma^{v}} \mid \theta_{i}\right)
$$

holds for all $\phi_{R}$, $\phi_{Q}$, and $n$ and for all possible realizations of $\theta$, at least weakly for $\theta_{Q}$-agents, and strictly for $\theta_{R}$-agents.

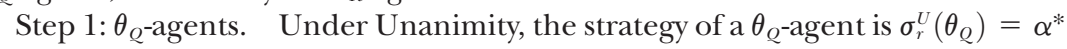
and $\sigma_{v}^{U}\left(\theta_{Q}\right)=1-\alpha^{*}$ (proposition 9 in app. B). As she is indifferent between playing $r$ and $v$, her interim utility is the same in both cases. If she plays $v$, then the decision is $Q$ for sure, and her expected utility is minus the probability to be in state $\omega_{R}$ conditional on being type $\theta_{Q}$, that is,

$$
u_{i}\left(U_{\sigma^{u}} \mid \theta_{Q}\right)=\frac{-1}{1+\phi_{Q}} .
$$

Under Veto, at the information-aggregation equilibrium (proposition 6 in

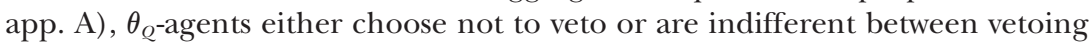
and voting $q$. They cannot be strictly worse off under $V_{\sigma^{u}}$ than $U_{\sigma^{v}}$ by a simple revealed preference argument: these agents could always ensure a level of expected utility equal to $-1 /\left(1+\phi_{Q}\right)$ by vetoing. Note that we can in fact show that they are strictly better off under $V_{\sigma^{u}}$ than $U_{\sigma^{v}}$ when they choose not to (but this is not needed for theorem 1). 


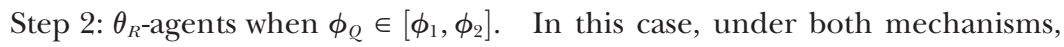
$\theta_{R}$-agents always play $r$. Their interim utility is (for any $\phi_{Q}$ )

$$
\begin{aligned}
& u_{i}\left(U_{\sigma^{v}} \mid \theta_{R}\right)=\frac{-\phi_{R}}{1+\phi_{R}}+\frac{\phi_{R} \operatorname{Pr}\left(x_{r}=n-1 \mid \omega_{R}, \sigma^{U}\right)-\operatorname{Pr}\left(x_{r}=n-1 \mid \omega_{Q}, \sigma^{U}\right)}{1+\phi_{R}}, \text { (C2) } \\
& u_{i}\left(V_{\sigma^{\mu}} \mid \theta_{R}\right)=\frac{-\phi_{R}}{1+\phi_{R}}+\frac{\phi_{R} \operatorname{Pr}\left(x_{r} \geq \frac{n-1}{2} \mid \omega_{R}, \sigma^{I A}\right)-\operatorname{Pr}\left(x_{r} \geq \frac{n-1}{2} \mid \omega_{Q}, \sigma^{I A}\right)}{1+\phi_{R}} .
\end{aligned}
$$

For condition (C1) to hold strictly, we need

$$
\begin{aligned}
& \phi_{R} \operatorname{Pr}\left(x_{r} \geq \frac{n-1}{2} \mid \omega_{R}, \sigma^{I A}\right)-\operatorname{Pr}\left(x_{r} \geq \frac{n-1}{2} \mid \omega_{Q}, \sigma^{I A}\right) \\
> & \phi_{R} \operatorname{Pr}\left(x_{r}=n-1 \mid \omega_{R}, \sigma^{U}\right)-\operatorname{Pr}\left(x_{r}=n-1 \mid \omega_{Q}, \sigma^{U}\right) .
\end{aligned}
$$

From propositions 6 and 9, this becomes

$$
\begin{gathered}
\sum_{j=(n-1) / 2}^{n-1}\left(\begin{array}{c}
n-1 \\
j
\end{array}\right) \frac{\phi_{R}\left(\phi_{R}\left(\phi_{Q}-1\right)\right)^{j}\left(\phi_{R}-1\right)^{n-1-j}-\left(\phi_{Q}-1\right)^{j}\left[\phi_{Q}\left(\phi_{R}-1\right)\right]^{n-1-j}}{\left(\phi_{Q} \phi_{R}-1\right)^{n-1}} \\
>\frac{\phi_{R}\left(\phi_{R}\left(\phi_{Q}-1\right)+\left(\phi_{R}-1\right) \alpha^{*}\right)^{n-1}-\left[\left(\phi_{Q}-1\right)+\phi_{Q}\left(\phi_{R}-1\right) \alpha^{*}\right]^{n-1}}{\left(\phi_{Q} \phi_{R}-1\right)^{n-1}} .
\end{gathered}
$$

We can then rewrite the left-hand side as

$$
\frac{\left(\phi_{R}-1\right)^{n-1}}{\left(\phi_{Q} \phi_{R}-1\right)^{n-1}} \sum_{j=(n-1) / 2}^{n-1}\left(\begin{array}{c}
n-1 \\
j
\end{array}\right)\left(\frac{\phi_{Q}-1}{\phi_{R}-1}\right)^{j}\left[\left(\phi_{R}\right)^{j+1}-\left(\phi_{Q}\right)^{n-1-j}\right] .
$$

We can also distribute the right-hand side and group it similarly to the left-hand side:

$$
\frac{\left(\phi_{R}-1\right)^{n-1}}{\left(\phi_{Q} \phi_{R}-1\right)^{n-1}} \sum_{0}^{n-1}\left(\begin{array}{c}
n-1 \\
j
\end{array}\right)\left(\frac{\phi_{Q}-1}{\phi_{R}-1}\right)^{j}\left(\alpha^{*}\right)^{n-1-j}\left[\left(\phi_{R}\right)^{j+1}-\left(\phi_{Q}\right)^{n-1-j}\right] .
$$

We therefore need to show

$$
\begin{gathered}
\sum_{j=(n-1) / 2}^{n-1}\left(\begin{array}{c}
n-1 \\
j
\end{array}\right)\left(\frac{\phi_{Q}-1}{\phi_{R}-1}\right)^{j}\left[\left(\phi_{R}\right)^{j+1}-\left(\phi_{Q}\right)^{n-1-j}\right] \\
>\sum_{0}^{n-1}\left(\begin{array}{c}
n-1 \\
j
\end{array}\right)\left(\frac{\phi_{Q}-1}{\phi_{R}-1}\right)^{j}\left(\alpha^{*}\right)^{n-1-j}\left[\left(\phi_{R}\right)^{j+1}-\left(\phi_{Q}\right)^{n-1-j}\right] .
\end{gathered}
$$

We can now compare these two sums term by term (recall that here $\phi_{Q} \in$ $\left.\left[\phi_{1}, \phi_{2}\right]\right)$ and show that the left-hand-side terms are always larger that the righthand-side terms. First, observe that the terms in $j=n-1$ cancel out. Second, for $j \in[(n-1) / 2, n-1)$, note that from $\phi_{Q} \leq \phi_{2} \equiv\left(\phi_{R}\right)^{(n+1) /(n-1)}$, we have that $\left(\phi_{R}\right)^{j+1} \geq\left(\phi_{Q}\right)^{n-1-j}$, with strict inequality for $j>(n-1) / 2$. Since $\alpha^{*}<1$, the left- 
hand-side terms are strictly higher than the right-hand-side terms for all $j \in$ $[(n-1) / 2, n-1)$. Third, for $j<(n-1) / 2$, note that from $\phi_{Q} \geq \phi_{1} \equiv\left(\phi_{R}\right)^{(n-1) /(n+1)}$, we have that $\left(\phi_{R}\right)^{j+1} \leq\left(\phi_{Q}\right)^{n-1-j}$, with strict inequality for $j<(n-3) / 2$. So all the respective terms on the right-hand side are negative and are equal to zero in the left-hand side.

Step 3: $\theta_{R}$-agents when $\phi_{Q} \in\left(\phi_{2}, \phi_{3}\right] . \quad$ In this case, under $U_{\sigma^{v}}, \theta_{R^{-}}$-agents still always play $r$. Hence, their interim utility is still given by (C2). But we have $\sigma_{r}^{I A}\left(\theta_{R}\right)=1-\varepsilon^{*}$ and $\sigma_{q}^{I A}\left(\theta_{R}\right)=\varepsilon^{*}$ (proposition 6). Since they mix, their interim utility is the same in both cases:

$$
u_{i}\left(V_{\sigma^{\text {IA }}} \mid \theta_{R}\right)=\frac{-\phi_{R}}{1+\phi_{R}}+\frac{\phi_{R} \operatorname{Pr}\left(x_{r} \geq \frac{n+1}{2} \mid \omega_{R}, \sigma^{I A}\right)-\operatorname{Pr}\left(x_{r} \geq \frac{n+1}{2} \mid \omega_{Q}, \sigma^{I A}\right)}{1+\phi_{R}} .
$$

This can be rewritten as follows:

$$
\begin{aligned}
u_{i}\left(V_{\sigma^{n}} \mid \theta_{R}\right)= & \left(\phi_{R} \phi_{Q}-1\right)^{1 /(n-1)} \sum_{j=(n+1) / 2}^{n-1}\left(\begin{array}{c}
n-1 \\
j
\end{array}\right)\left(1-\varepsilon^{*}\right)^{j}\left(\phi_{Q}-1\right)^{j} \\
& \times\left\{\left(\phi_{R}\right)^{j+1}\left[\varepsilon^{*} \phi_{R}\left(\phi_{Q}-1\right)+\left(\phi_{R}-1\right)\right]^{n-1-j}\right. \\
& \left.-\left[\varepsilon^{*}\left(\phi_{Q}-1\right)+\phi_{Q}\left(\phi_{R}-1\right)\right]^{n-1-j}\right\} .
\end{aligned}
$$

By the definition of $\varepsilon^{*}$, it is easy to show that the term in $j=(n-1) / 2$ is nil (it exactly corresponds to the agent's gain of playing $r$, which is nil in this equilibrium) and that the terms in $j<(n-1) / 2$ are strictly negative. Therefore, we have that

$$
\begin{aligned}
u_{i}\left(V_{\sigma^{n}} \mid \theta_{R}\right)> & \left(\phi_{R} \phi_{Q}-1\right)^{1 /(n-1)} \sum_{j=0}^{n-1}\left(\begin{array}{c}
n-1 \\
j
\end{array}\right)\left(1-\varepsilon^{*}\right)^{j}\left(\phi_{Q}-1\right)^{j} \\
& \times\left\{\left(\phi_{R}\right)^{j+1}\left[\varepsilon^{*} \phi_{R}\left(\phi_{Q}-1\right)+\left(\phi_{R}-1\right)\right]^{n-1-j}\right. \\
& \left.-\left[\varepsilon^{*}\left(\phi_{Q}-1\right)+\phi_{Q}\left(\phi_{R}-1\right)\right]^{n-1-j}\right\} .
\end{aligned}
$$

Using the binomial theorem, this corresponds to

$$
\begin{aligned}
u_{i}\left(V_{\sigma^{n}} \mid \theta_{R}\right)> & \left(\phi_{R} \phi_{Q}-1\right)^{1 /(n-1)}\left\{\phi_{R}\left[\phi_{R}\left(\phi_{Q}-1\right)+\left(\phi_{R}-1\right)\right]^{n-1}\right. \\
& \left.-\left[\left(\phi_{Q}-1\right)+\phi_{Q}\left(\phi_{R}-1\right)\right]^{n-1}\right\} .
\end{aligned}
$$

Now, from proposition 9 , we have

$$
\begin{aligned}
u_{i}\left(U_{\sigma^{v}} \mid \theta_{R}\right)= & \left(\phi_{R} \phi_{Q}-1\right)^{1 /(n-1)}\left\{\phi_{R}\left[\phi_{R} \phi_{Q}-1\right)+\left(\phi_{R}-1\right) \alpha^{*}\right]^{n-1} \\
& \left.-\left[\left(\phi_{Q}-1\right)+\phi_{Q}\left(\phi_{R}-1\right) \alpha^{*}\right]^{n-1}\right\} .
\end{aligned}
$$

Thus, for $u_{i}\left(V_{\sigma^{\mu}} \mid \theta_{R}\right)>u_{i}\left(U_{\sigma^{v}} \mid \theta_{R}\right)$ to hold, it is sufficient that

$$
\begin{gathered}
\phi_{R}\left[\phi_{R}\left(\phi_{Q}-1\right)+\left(\phi_{R}-1\right)\right]^{n-1}-\left[\left(\phi_{Q}-1\right)+\phi_{Q}\left(\phi_{R}-1\right)\right]^{n-1} \\
\geq \phi_{R}\left[\phi_{R}\left(\phi_{Q}-1\right)+\left(\phi_{R}-1\right) \alpha^{*}\right]^{n-1}-\left[\left(\phi_{Q}-1\right)+\phi_{Q}\left(\phi_{R}-1\right) \alpha^{*}\right]^{n-1} .
\end{gathered}
$$

To prove that this is always satisfied, we show that the derivative of the right-hand side with respect to $\alpha^{*}$ is positive: 


$$
\begin{gathered}
(n-1)\left(\phi_{R}-1\right)\left\{\phi_{R}\left[\phi_{R}\left(\phi_{Q}-1\right)+\left(\phi_{R}-1\right) \alpha^{*}\right]^{n-2}\right. \\
\left.-\phi_{Q}\left[\left(\phi_{Q}-1\right)+\phi_{Q}\left(\phi_{R}-1\right) \alpha^{*}\right]^{n-2}\right\}>0 .
\end{gathered}
$$

To see this, we use that $G^{U}\left(v \mid \theta_{Q}\right)=0$ in equilibrium requires (see the proof of proposition 6 in the online appendix)

$$
\phi_{R}\left(\phi_{Q}-1\right)+\left(\phi_{R}-1\right) \alpha^{*}=\phi_{Q}^{1 /(n-1)}\left[\left(\phi_{Q}-1\right)+\phi_{Q}\left(\phi_{R}-1\right) \alpha^{*}\right] .
$$

Then this condition boils down to

$$
\phi_{R} \phi_{Q}^{-1 /(n-1)}>1,
$$

which is always strictly satisfied when $\phi_{Q}<\phi_{4}$.

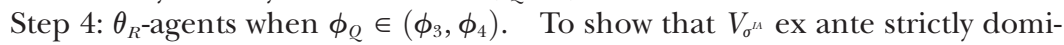
nates $U_{\sigma^{v}}$, we construct a (nonequilibrium) strategy profile $\sigma^{\prime}$ such that $V_{\sigma^{\prime}}$ ex ante dominates $V_{\sigma^{v}}$. That is,

$$
u_{i}\left(V_{\sigma^{\prime}}\right)>u_{i}\left(V_{\sigma^{u}}\right) .
$$

By McLennan (1998), which shows that the strategy that maximizes ex ante welfare in a common-value game must be an equilibrium, we know that there must be another equilibrium under Veto that ex ante strictly dominates $\sigma^{U}$. Since there are only two equilibria under Veto, this dominating equilibrium must be $\sigma^{I A}$. Since $V_{\sigma^{v}}$ is equivalent to $U_{\sigma^{v}}$, that is, $V_{\sigma^{v}}(\boldsymbol{\theta})=U_{\sigma^{v}}(\boldsymbol{\theta})$ for all $\boldsymbol{\theta}$, we have that

$$
u_{i}\left(V_{\sigma^{u}}\right)>u_{i}\left(U_{\sigma^{u}}\right) .
$$

Define $\sigma^{\prime}$ as follows:

$$
\sigma_{r}^{\prime}\left(\theta_{R}\right)=1, \quad \sigma_{q}^{\prime}\left(\theta_{R}\right)=\alpha^{*}, \quad \sigma_{v}^{\prime}\left(\theta_{R}\right)=1-\alpha^{*},
$$

where $\alpha^{*}$ corresponds to that of proposition 7 .

The $\theta_{R}$-agents are strictly better off if

$$
\begin{gathered}
\sum_{j=(n-1) / 2}^{n-1}\left(\begin{array}{c}
n-1 \\
j
\end{array}\right) \frac{\phi_{R}\left(\phi_{R}\left(\phi_{Q}-1\right)\right)^{j}\left[\alpha^{*}\left(\phi_{R}-1\right)\right]^{n-1-j}-\left(\phi_{Q}-1\right)^{j}\left[\alpha^{*} \phi_{Q}\left(\phi_{R}-1\right)\right]^{n-1-j}}{\left(\phi_{Q} \phi_{R}-1\right)^{n-1}} \\
>\frac{\phi_{R}\left(\phi_{R}\left(\phi_{Q}-1\right)+\left(\phi_{R}-1\right) \alpha^{*}\right)^{n-1}-\left[\left(\phi_{Q}-1\right)+\phi_{Q}\left(\phi_{R}-1\right) \alpha^{*}\right]^{n-1}}{\left(\phi_{Q} \phi_{R}-1\right)^{n-1}} .
\end{gathered}
$$

We can then rewrite the left-hand side as

$$
\frac{\left(\phi_{R}-1\right)^{n-1}}{\left(\phi_{Q} \phi_{R}-1\right)^{n-1}} \sum_{j=(n-1) / 2}^{n-1}\left(\begin{array}{c}
n-1 \\
j
\end{array}\right)\left(\frac{\phi_{Q}-1}{\phi_{R}-1}\right)^{j}\left(\alpha^{*}\right)^{n-1-j}\left[\left(\phi_{R}\right)^{j+1}-\left(\phi_{Q}\right)^{n-1-j}\right] .
$$

We can also distribute the right-hand side and group it similarly to the left-hand side:

$$
\frac{\left(\phi_{R}-1\right)^{n-1}}{\left(\phi_{Q} \phi_{R}-1\right)^{n-1}} \sum_{0}^{n-1}\left(\begin{array}{c}
n-1 \\
j
\end{array}\right)\left(\frac{\phi_{Q}-1}{\phi_{R}-1}\right)^{j}\left(\alpha^{*}\right)^{n-1-j}\left[\left(\phi_{R}\right)^{j+1}-\left(\phi_{Q}\right)^{n-1-j}\right] .
$$


And it is obvious that the condition holds strictly since we have that $\left(\phi_{R}\right)^{j+1} \leq$ $\left(\phi_{Q}\right)^{n-1-j}$ for all $j \leq(n-3) / 2$ since $\phi_{Q} \geq \phi_{3} \equiv\left(\phi_{R}\right)^{(n+1) /(n-1)}$ (see the proof for proposition 6).

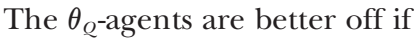

$$
\begin{gathered}
\sum_{j=(n-1) / 2}^{n-1}\left(\begin{array}{c}
n-1 \\
j
\end{array}\right) \frac{\left[\phi_{R}\left(\phi_{Q}-1\right)\right]^{j}\left[\alpha^{*}\left(\phi_{R}-1\right)\right]^{n-1-j}-\phi_{Q}\left(\phi_{Q}-1\right)^{j}\left[\alpha^{*} \phi_{Q}\left(\phi_{R}-1\right)\right]^{n-1-j}}{\left(\phi_{Q} \phi_{R}-1\right)^{n-1}} \\
\quad \geq \frac{\left[\phi_{R}\left(\phi_{Q}-1\right)+\left(\phi_{R}-1\right) \alpha^{*}\right]^{n-1}-\phi_{Q}\left(\left(\phi_{Q}-1\right)+\phi_{Q}\left(\phi_{R}-1\right) \alpha^{*}\right)^{n-1}}{\left(\phi_{Q} \phi_{R}-1\right)^{n-1}} .
\end{gathered}
$$

We can then rewrite the left-hand side as

$$
\frac{\left(\phi_{R}-1\right)^{n-1}}{\left(\phi_{Q} \phi_{R}-1\right)^{n-1}} \sum_{j=(n+1) / 2}^{n-1}\left(\begin{array}{c}
n-1 \\
j
\end{array}\right)\left(\frac{\phi_{Q}-1}{\phi_{R}-1}\right)^{j}\left(\alpha^{*}\right)^{n-1-j}\left[\left(\phi_{R}\right)^{j}-\left(\phi_{Q}\right)^{n-j}\right] .
$$

We can also distribute the right-hand side and group it similarly to the left-hand side:

$$
\frac{\left(\phi_{R}-1\right)^{n-1}}{\left(\phi_{Q} \phi_{R}-1\right)^{n-1}} \sum_{0}^{n-1}\left(\begin{array}{c}
n-1 \\
j
\end{array}\right)\left(\frac{\phi_{Q}-1}{\phi_{R}-1}\right)^{j}\left(\alpha^{*}\right)^{n-1-j}\left[\left(\phi_{R}\right)^{j}-\left(\phi_{Q}\right)^{n-j}\right] .
$$

And it is obvious that the condition holds since we have that $\left(\phi_{R}\right)^{j} \leq\left(\phi_{Q}\right)^{n-j}$ for all $j \leq(n-1) / 2$ since $\phi_{Q} \geq \phi_{3} \equiv\left(\phi_{R}\right)^{(n+1) /(n-1)}$. Hence, $u_{i}\left(V_{\sigma^{\prime}}\right)>u_{i}\left(V_{\sigma^{v}}\right)$, which is what we need.

Step 5: $\theta_{R}$-agents when $\phi_{Q} \in\left(1, \phi_{1}\right)$. First we use $\eta^{*}$ and $\alpha^{*}$ (from propositions 6 and 9 ) to derive the probabilities that the reform is adopted (conditional on the agent being $\left.\theta_{R}\right)$ in each state under both rules (remember that $\sigma_{r}^{A I}\left(\theta_{R}\right)=$ $1=\sigma_{r}^{U}\left(\theta_{R}\right)$ in the case under consideration):

$$
\begin{aligned}
\Omega^{V}\left(\omega_{R}\right) & \equiv \operatorname{Pr}\left(V_{\sigma^{u}}(\theta)=R \mid \omega_{R}\right)=\operatorname{Pr}\left(x_{r} \geq \frac{n-1}{2} \text { and } x_{v}=0 \mid \omega_{R}, \sigma^{I A}\right) \\
& =\frac{1}{\left[\left(\phi_{Q}\right)^{2 n /(n-1)}-1\right]^{n-1}} \sum_{j=(n-1) / 2}^{n-1}\left(\begin{array}{c}
n-1 \\
j
\end{array}\right)\left(\phi_{Q}\right)^{j(n+1) /(n-1)}\left\{\left(\phi_{Q}-1\right)^{j}\left[\left(\phi_{Q}\right)^{(n+1) /(n-1)}-1\right]^{n-1-j}\right\} \\
\Omega^{V}\left(\omega_{Q}\right) & \equiv \operatorname{Pr}\left(V_{\sigma^{u}}(\theta)=R \mid \omega_{Q}\right)=\operatorname{Pr}\left(x_{r} \geq \frac{n-1}{2} \text { and } x_{v}=0 \mid \omega_{Q}, \sigma^{I A}\right) \\
& =\frac{1}{\left[\left(\phi_{Q}\right)^{2 n /(n-1)}-1\right]^{n-1}} \sum_{j=(n-1) / 2}^{n-1}\left(\begin{array}{c}
n-1 \\
j
\end{array}\right)\left(\phi_{Q}\right)^{n-1-j}\left\{\left(\phi_{Q}-1\right)^{j}\left[\left(\phi_{Q}\right)^{(n+1) /(n-1)}-1\right]^{n-1-j}\right\} \\
\Omega^{U}\left(\omega_{R}\right) & \equiv \operatorname{Pr}\left(U_{\sigma^{u}}(\theta)=R \mid \omega_{R}\right)=\operatorname{Pr}\left(x_{r}=n-1 \mid \omega_{R}, \sigma^{U}\right)=\phi_{Q} \frac{\left(\phi_{Q}-1\right)^{n-1}}{\left[\left(\phi_{Q}\right)^{n /(n-1)}-1\right]^{n-1}}, \\
\Omega^{U}\left(\omega_{Q}\right) & \equiv \operatorname{Pr}\left(U_{\sigma^{u}}(\theta)=R \mid \omega_{Q}\right)=\operatorname{Pr}\left(x_{r}=n-1 \mid \omega_{Q}, \sigma^{U}\right)=\frac{\left(\phi_{Q}-1\right)^{n-1}}{\left[\left(\phi_{Q}\right)^{n /(n-1)}-1\right]^{n-1}} .
\end{aligned}
$$

Note that they are all independent from $\phi_{R}$.

The interim utility of a $\theta_{R^{-}}$-agent is given by (C2) and (C3) under $U_{\sigma^{v}}$ and $V_{\sigma^{\mu}}$, respectively. Therefore, $u_{i}\left(V_{\sigma^{u}} \mid \theta_{R}\right)-u_{i}\left(U_{\sigma^{u}} \mid \theta_{R}\right)$ is given by 


$$
\frac{\phi_{R} \Omega^{V}\left(\omega_{R}\right)-\Omega^{V}\left(\omega_{Q}\right)}{1+\phi_{R}}-\frac{\phi_{R} \Omega^{U}\left(\omega_{R}\right)-\Omega^{U}\left(\omega_{Q}\right)}{1+\phi_{R}} .
$$

From step 2, we know that $u_{i}\left(V_{\sigma^{u}} \mid \theta_{R}\right)>u_{i}\left(U_{\sigma^{u}} \mid \theta_{R}\right)$ when $\phi_{Q}=\phi_{1}$. To prove that this inequality holds for any $\phi_{Q} \in\left(1, \phi_{R}\right)$, it is sufficient to prove that it is satisfied for any $\phi_{R}$ larger than the $\phi_{R}$ such that $\phi_{Q}=\phi_{1}$. Given that $\Omega^{\Psi}(\omega)$ is independent of $\phi_{R}$ for all $\Psi$ and $\omega$, a sufficient condition for $u_{i}\left(V_{\sigma^{\mu \prime}} \mid \theta_{R}\right)>u_{i}\left(U_{\sigma^{v}} \mid \theta_{R}\right)$ to be satisfied for larger $\phi_{R}$ is that $\Omega^{V}\left(\omega_{R}\right) \geq \Omega^{U}\left(\omega_{R}\right)$. Thus, we need to prove that the following inequality is satisfied when $\phi_{Q} \in\left(1, \phi_{1}\right)$ :

$$
\begin{aligned}
\sum_{j=(n-1) / 2}^{n-1}\left(\begin{array}{c}
n-1 \\
j
\end{array}\right) & \frac{\left[\left(\phi_{Q}-1\right) \phi_{Q}^{(n+1) /(n-1)}\right]^{j}\left[\phi_{Q}^{(n+1) /(n-1)}-1\right]^{n-1-j}}{\left[\phi_{Q}^{2 n /(n-1)}-1\right]^{n-1}} \\
& >\phi_{Q} \frac{\left(\phi_{Q}-1\right)^{n-1}}{\left[\left(\phi_{Q}\right)^{n /(n-1)}-1\right]^{n-1}} .
\end{aligned}
$$

This boils down to

$$
\begin{gathered}
\sum_{j=(n-1) / 2}^{n-1}\left(\begin{array}{c}
n-1 \\
j
\end{array}\right)\left[\left(\phi_{Q}-1\right) \phi_{Q}^{(n+1) /(n-1)}\right]^{j}\left[\phi_{Q}^{(n+1) /(n-1)}-1\right]^{n-1-j} \\
>\phi_{Q}\left(\left(\phi_{Q}\right)^{n /(n-1)}+1\right)^{n-1}\left(\phi_{Q}-1\right)^{n-1} .
\end{gathered}
$$

Using lemma 4 (see below), we can substitute for $\phi_{Q}^{(n+1) /(n-1)}-1$ and cancel the terms in $\left(\phi_{Q}-1\right)^{n-1}$. This gives

$$
\sum_{j=(n-1) / 2}^{n-1}\left(\begin{array}{c}
n-1 \\
j
\end{array}\right) \phi_{Q}^{j(n+1) /(n-1)}\left[\frac{n+1}{n-1} \phi_{Q}^{1 /(n-1)}\right]^{n-1-j}>\phi_{Q}\left(\left(\phi_{Q}\right)^{n /(n-1)}+1\right)^{n-1} .
$$

Using the binomial theorem, we have

$$
\sum_{j=(n-1) / 2}^{n-1}\left(\begin{array}{c}
n-1 \\
j
\end{array}\right) \phi_{Q}^{j[n /(n-1)]}\left(\frac{n+1}{n-1}\right)^{n-1-j}>\sum_{k=0}^{n-1}\left(\begin{array}{c}
n-1 \\
k
\end{array}\right)\left(\phi_{Q}\right)^{k[n /(n-1)]} .
$$

Given that the terms in $j=n-1=k$ cancel out, we can focus on $j<n-1$ and $k<n-1$. Note that, for all $j<n-1$,

$$
\left(\frac{n+1}{n-1}\right)^{n-1-j}=\left(1+\frac{2}{n-1}\right)^{n-1-j}=\left[1+\frac{\frac{2(n-1-j)}{n-1}}{n-1-j}\right]^{n-1-j} \geq 1+\frac{n-1-j}{\frac{n-1}{2}}
$$

(indeed, for $x \geq-1$ and $r \in \mathbb{R} \backslash(0,1)$, we know that $(1+x)^{r} \geq 1+r x$ is satisfied). Thus, it is sufficient to show that

$$
\sum_{j=(n-1) / 2}^{n-2}\left(\begin{array}{c}
n-1 \\
j
\end{array}\right) \phi_{Q}^{j[n /(n-1)]}\left(1+\frac{n-1-j}{\frac{n-1}{2}}\right)>\sum_{k=0}^{n-2}\left(\begin{array}{c}
n-1 \\
k
\end{array}\right)\left(\phi_{Q}\right)^{k[n /(n-1)]},
$$

or 


$$
\begin{aligned}
& \sum_{j=(n-1) / 2}^{n-2} \frac{(n-1) !}{(n-1-j) ! j !}\left(\frac{n-1-j}{\frac{n-1}{2}}\right)\left(\phi_{Q}\right)^{j[n /(n-1)]} \\
& >\sum_{k=0}^{(n-3) / 2} \frac{(n-1) !}{(n-1-k) ! k !}\left(\phi_{Q}\right)^{k[n /(n-1)]} .
\end{aligned}
$$

Let us compare the terms two-by-two in the following order: $j=(n-1) / 2$ with $k=(n-3) / 2, j=(n+1) / 2$ with $k=(n-5) / 2, j=(n+3) / 2$ with $k=$ $(n-7) / 2, \ldots$, and $j=n-2$ with $k=0$. This comparison boils down to

$$
\frac{n-1-j}{\frac{n-1}{2}} \frac{(n-1) !}{(n-1-j) ! j !} \geq \frac{(n-1) !}{(j+1) !(n-1-j-1) !} .
$$

Simple algebra gives $j \geq[(n-1) / 2]-1$, which is satisfied since we consider all

$$
j \in\left\{\frac{n-1}{2}, \frac{n+1}{2}, \ldots, n-2\right\} .
$$

QED

Lemma 4. For all $x \geq 1, n>1$, we have that

$$
x^{(n+1) /(n-1)}-1 \geq \frac{n+1}{n-1}\left[x^{n /(n-1)}-x^{1 /(n-1)}\right] .
$$

Proof. See the online appendix.

\section{References}

Aghion, P., and P. Bolton. 2003. "Incomplete Social Contracts." LEuropean Econ. Assoc. 1 (1): 38-67.

Ahn, D. S., and S. Oliveros. 2012. "Combinatorial Voting." Econometrica 80 (1): 89-141.

2014. "The Condorcet Jur(ies) Theorem.” I. Econ. Theorv 150:841-51.

Austen-Smith, D., and J. Banks. 1996. "Information Aggregation, Rationality, and the Condorcet Jury Theorem." American Polit. Sci. Rev. 90:34-45.

Austen-Smith, D., and T. Feddersen. 2006. "Deliberation, Preference Uncertainty and Voting Rules." American Polit. Sci. Rev. 100 (2): 209-17.

Battaglini, M., R. Morton, and T. Palfrey. 2008. "Information Aggregation and Strategic Abstention in Large Laboratory Elections." A.E.R. Papers and Proc. 98 (2): 194-200.

2010. "The Swing Voter's Curse in the Laboratory." Rev. Econ. Studies 77:61-89.

Bhattacharya, S. 2013. "Preference Monotonicity and Information Aggregation in Elections." Econometrica 81 (3): 1229-47.

Blake, D. J., and A. Lockwood Payton. 2015. "Balancing Design Objectives: Analyzing New Data on Voting Rules in Intergovernmental Organizations." Rev. Internat. Org. 10 (3): 377-402.

Bond, P., and H. Eraslan. 2009. "Strategic Voting over Strategic Proposals." Rev. Econ. Studies 77 (2): 459-90.

Bouton, L. 2013. "A Theory of Strategic Voting in Runoff Elections." A.E.R. 103 (4): $1248-88$. 
Bouton, L., and M. Castanheira. 2012. "One Person, Many Votes: Divided Majority and Information Aggregation.” Econometrica 80 (1): 43-87.

Bouton, L., M. Castanheira, and A. Llorente-Saguer. 2016. "Divided Majority and Information Aggregation: Theory and Experiment." I. Public Econ. 134:114-28.

Bouton, L., A. Llorente-Saguer, and F. Malherbe. 2017. "Unanimous Rules in the Laboratory." Games and Econ. Behavior 102:179-98.

Buchanan, J. M., and G. Tullock. 1962. The Calculus of Consent. Ann Arbor: Univ. Michigan Press.

Chen, Y., and P. Ordeshook. 1998. "Veto Games: Spatial Committees under Unanimity Rule." Public Choice 97 (4): 617-43.

Chwe, M. 1999. "Minority Voting Rights Can Maximize Majority Welfare." American Polit. Sci. Rev. 93 (1): 85-97.

Cogen, M. 2015. An Introduction to European International Organizations. Farnham, UK: Ashgate.

Costinot, A., and N. Kartik. 2007. "On Optimal Voting Rules under Homogenous Preferences.” Manuscript, Dept. Econ., Univ. California San Diego.

Coughlan, P. 2000. "In Defense of Unanimous Jury Verdicts: Mistrials, Communication and Strategic Voting." American Polit. Sci. Rev. 94:375-93.

Dasgupta, P., and E. Maskin. 2008. "On the Robustness of Majority Rule." L. European Econ. Assoc. 6 (5): 949-73.

Delbruck, J. [1977] 1996. "Peacekeeping by the United Nations and the Rule of Law." Reprinted in Die Konstitution des Friedens als Rechtsordnung. Berlin: Duncker \& Humblot.

Dewan, T., and D. Myatt. 2007. "Leading the Party: Coordination, Direction, and Communication." American Polit. Sci. Rev. 101:825-43.

Diamond, S., M. Rose, and B. Murphy. 2006. "Revisiting the Unanimity Requirement: The Behavior of the Non-unanimous Civil Jury." Northwestern Univ. Law Rev. 100 (1): 201-30.

Dougherty, K., and J. Edward. 2005. "A Nonequilibrium Analysis of Unanimity Rule, Majority Rule, and Pareto." Econ. Inquiry 43 (4): 855-64.

Duggan, J., and C. Martinelli. 2001. "A Bayesian Model of Voting in Juries." Games and Econ. Behavior 37 (2): 259-94.

Dziuda, W., and A. Loeper. 2016. "Dynamic Collective Choice with Endogenous Status Quo.” I.P.E. 124 (4): 1148-86.

Ekmekci, M., and S. Lauermann. 2015. "Manipulated Electorates and Information Aggregation.” Manuscript, Dept. Econ., Univ. Bonn.

Fassbender, B. 1998. UN Security Council Reform and the Right of Veto: A Constitutional Perspective. The Hague: Kluwer Law Internat.

Feddersen, T., and W. Pesendorfer. 1996. "The Swing Voter's Curse." A.E.R. $86: 408-24$

_. 1997. "Voting Behavior and Information Aggregation in Elections with Private Information.” Econometrica 65:1029-58.

- 1998. "Convicting the Innocent: The Inferiority of Unanimous Jury Verdicts." American Polit. Sci. Rev. 92:23-35.

Felsenthal, Dan S., and M. Machover. 2001. "Models and Reality: The Curious Case of the Absent Abstention." In Power Indices and Coalition Formation, edited by J. M. Holler and G. Owen, 87-104. Dordrecht: Kluwer Academic.

Gerardi, D. 2000. "Jury Verdicts and Preference Diversity." American Polit. Sci. Rev. 94 (2): 395-406.

Gerardi, D., and L. Yariv. 2007. “Deliberative Voting.” J. Econ. Theory 114:357-94.

Goeree, J., and L. Yariv. 2011. "An Experimental Study of Collective Deliberation." Econometrica 79 (3): 893-921. 
Groseclose, T., and N. McCarty. 2001. "The Politics of Blame: Bargaining before an Audience." American I. Polit. Sci. 45 (1): 100-119.

Gross, L. 1951. "Voting in the Security Council: Abstention from Voting and Absence from Meetings." Yale I. Law 60 (2): 209-57.

Guarnaschelli, S., R. McKelvey, and T. Palfrey. 2000. "An Experimental Study of Jury Decision Rules.” American Polit. Sci. Rev. 94 (2): 407-23.

Guttman, J. 1998. "Unanimity and Majority Rule: The Calculus of Consent Reconsidered." European I. Polit. Econ. 14:189-207.

Herrera, H., J. McMurray, and A. Llorente-Saguer. 2015. “The Marginal Voter's Curse." Manuscript, School Econ. and Finance, Queen Mary Univ. London.

Holmstrom, B., and R. Myerson. 1983. "Efficient and Durable Decision Rules with Incomplete Information." Econometrica 51 (6): 1799-1819.

Koremenos, B. 2013. "The Continent of International Law." L. Conflict Resolution 57 (4): $653-81$.

Lee, D. 1947. "The Genesis of the Veto." Internat. Org. 1 (1): 33-42.

Liang, Y.-L. 1950. "Abstention and Absence of a Permanent Member in Relation to the Voting Procedure in the Security Council." American I. Internat. Law 44 (4): 694-708.

Maggi, G., and M. Morelli. 2006. "Self-Enforcing Voting in International Organizations." A.E.R. 96 (4): 1137-58.

Mandler, M. 2012. "The Fragility of Information Aggregation in Large Elections." Games and Econ. Behavior 74 (1): 257-68.

Martinelli, C. 2006. "Would Rational Voters Acquire Costly Information?” I. Econ. Theory 129:225-51.

Maug, E., and B. Yilmaz. 2002. "Two-Class Voting: A Mechanism for Conflict Resolution." A.E.R. 92 (5): 1448-71.

May, Kenneth O. 1952. "A Set of Independent Necessary and Sufficient Conditions for Simple Majority Decisions." Econometrica 20:680-84.

McGann, A. J. 2004. "The Tyranny of the Supermajority: How Majority Rule Protects Minorities." L. Theoretical Politics 16 (1): 53-77.

McLennan, A. 1998. "Consequences of the Condorcet Jury Theorem for Beneficial Information Aggregation by Rational Agents." American Polit. Sci. Rev. 92: 413-18.

McMurray, J. 2013. "Aggregating Information by Voting: The Wisdom of the Experts versus the Wisdom of the Masses." Rev. Econ. Studies 80 (1): 277-312.

Myatt, D. 2007. "On the Theory of Strategic Voting." Rev. Econ. Studies 74:255-81. _. 2015. "A Theory of Voter Turnout." Manuscript, London Bus. School.

Myerson, R. 1998. "Population Uncertainty and Poisson Games." Internat. I. Game Theorv 27:375-92.

. 2000. "Large Poisson Games." L. Econ. Theory 94:7-45.

2002. "Comparison of Scoring Rules in Poisson Voting Games." L.Econ. Theory 103:219-51.

Myerson, R., and R. Weber. 1993. "A Theory of Voting Equilibria.” American Polit. Sci. Rev. 87:102-14.

Neilson, W., and H. Winter. 2005. "The Elimination of Hung Juries: Retrials and Nonunanimous Verdicts." Internat. Rev. Law and Econ. 25:1-19.

Nunnari, S. 2016. "Dynamic Legislative Bargaining with Veto Power: Theory and Experiments." Manuscript, Dept. Econ., Bocconi Univ.

Persico, N. 2004. "Committee Design with Endogenous Information.” Rev. Econ. Studies 71:165-91.

Piketty, T. 2000. "Voting as Communicating." Rev. Econ. Studies 67:169-91. 
Posner, E., and A. Sykes. 2014. "Voting Rules and International Organizations." Coase-Sandor Working Paper Series in Law and Economics, no. 673, Law School, Univ. Chicago.

Reston, J. 1946. "Votes and Vetoes." Foreion Affairs 26:13-22.

Romme, A. G. L. 2004. "Unanimity Rule and Organizational Decision Making: A Simulation Model." Org. Sci. 15 (6): 704-18.

Sieberson, S. 2010. "Inching toward EU Supranationalism? Qualified Majority Voting and Unanimity under the Treaty of Lisbon." Virginia J. Internat. Law 50 (4): 919-95.

Sievers, L., and S. Daws. 2014. The Procedure of the UN Security Council. 4th ed. Oxford: Oxford Univ. Press.

Sobel, R., and R. Holcombe. 2001. "The Unanimous Voting Rule Is Not the Political Equivalent to Market Exchange." Public Choice 106:233-42.

Stavropoulos, C. 1967. "The Practice of Voluntary Abstentions by Permanent Members of the Security Council under Article 27, Paragraph 3, of the Charter of the United Nations." American I. Internat. Law 61 (3): 737-52.

Truman, H. 1965. Year of Decisions. New York: New American Library.

Tsebelis, G. 2002. Veto Plavers: How Political Institutions Work. Princeton, NJ: Princeton Univ. Press.

Van Weelden, R. 2008. "Deliberation Rules and Voting." O. I. Polit. Sci. 3:83-88.

Wicksell, K. [1896] 1967. "A New Principle of Just Taxation." In Classics in the Theory of Public Finance, edited by R. A. Musgrave and A. T. Peacocks, 72-118. New York: St. Martin's.

Wilcox, F. 1945. "The Yalta Voting Formula." American Polit. Sci. Rev. 39 (5): 94356.

Winter, E. 1996. "Voting and Vetoing." American Polit. Sci. Rev. 90 (4): 813-23.

Zamora, S. 1980. "Voting in International Economic Organizations." American I. Internat. Law 74 (3): 566-608. 